\title{
Magnetic Field Promotes Migration of Schwann Cells with Chondroitinase ABC (ChABC)-Loaded Superparamagnetic Nanoparticles Across Astrocyte Boundary in vitro
}

This article was published in the following Dove Press journal:

International Journal of Nanomedicine

\author{
Jianbo Gao, (D)' Bing Xia,' \\ Shengyou Li, (iD)' \\ Liangliang Huang, ${ }^{2}$ Teng Ma,' \\ Xiaowei Shi, (D) ${ }^{1}$ Kai Luo, (iD) ${ }^{3}$ \\ Yujie Yang,' Laihe Zhao,' \\ Hao Zhang, ${ }^{4}$ Beier Luo, (iD ${ }^{5}$ \\ Jinghui Huang (iD) \\ 'Department of Orthopaedics, Xijing \\ Hospital, Fourth Military Medical \\ University, Xi'an, People's Republic of \\ China; ${ }^{2}$ Department of Orthopaedics, \\ The General Hospital of Central Theater \\ Command of People's Liberation Army, \\ Wuhan, People's Republic of China; \\ ${ }^{3}$ Department of Orthopaedics, The \\ 985th Hospital of the PLA Joint Logistics \\ Support Force, Taiyuan, People's Republic \\ of China; ${ }^{4}$ Department of Spinal Surgery, \\ People's Hospital of Longhua District, \\ Shenzhen, People's Republic of China; \\ ${ }^{5}$ Department of Orthopaedics, Changhai \\ Hospital, Second Military Medical \\ University, Shanghai, People's Republic of \\ China
}

*These authors contributed equally to this work

Correspondence: Beier Luo

Department of Orthopaedics, Changhai

Hospital, Second Military Medical

University, 800 Xiangyin Road, Shanghai

200433, People's Republic of China

$\mathrm{Tel} / \mathrm{Fax}+862131161700$

Email bear_luo@I26.com

Jinghui Huang

Department of Orthopaedics, Xijing

Hospital, Fourth Military Medical

University, 169 Changle West Road,

Xi'an, Shaanxi 710032, People's Republic

of China

Tel/Fax +862984775275

Email huangjh@fmmu.edu.cn
Purpose: The clinical outcome of spinal cord injury is usually poor due to the lack of axonal regeneration and glia scar formation. As one of the most classical supporting cells in neural regeneration, Schwann cells (SCs) provide bioactive substrates for axonal migration and release molecules that regulate axonal growth. However, the effect of SC transplantation is limited by their poor migration capacity in the astrocyte-rich central nervous system.

Methods: In this study, we first magnetofected SCs with chondroitinase ABCpolyethylenimine functionalized superparamagnetic iron oxide nanoparticles (ChABC/PEISPIONs) to induce overexpression of ChABC for the removal of chondroitin sulfate proteoglycans. These are inhibitory factors and forming a dense scar that acts as a barrier to the regenerating axons. In vitro, we observed the migration of SCs in the region of astrocytes after the application of a stable external magnetic field.

Results: We found that magnetofection with $\mathrm{ChABC/PEI-SPIONs} \mathrm{significantly} \mathrm{up-regulated} \mathrm{the}$ expression of $\mathrm{ChABC}$ in SCs. Under the driven effect of the directional magnetic field (MF), the migration of magnetofected SCs was enhanced in the direction of the magnetic force. The number of SCs with ChABC/PEI-SPIONs migrated and the distance of migration into the astrocyte region was significantly increased. The number of SCs with ChABC/PEI-SPIONs that migrated into the astrocyte region was 11.6- and 4.6-fold higher than those observed for the intact control and nonMF groups, respectively. Furthermore, it was found that SCs with ChABC/PEI-SPIONs were in close contact with astrocytes and no longer formed boundaries in the presence of MF.

Conclusion: The mobility of the SCs with ChABC/PEI-SPIONs was enhanced along the axis of MF, holding the potential to promote nerve regeneration by providing a bioactive microenvironment and relieving glial obstruction to axonal regeneration in the treatment of spinal cord injury.

Keywords: Schwann cells, astrocytes, magnetic field, superparamagnetic iron oxide nanoparticles, spinal cord injury, cell orientation

\section{Introduction}

The repair of spinal cord injury has traditionally been a challenge in clinics. ${ }^{1,2}$ In contrast to the intrinsic regenerative properties of axons in the peripheral nervous system (PNS), axons in the central nervous system (CNS) exhibit poor regeneration. The regenerative properties of PNS neurons are partially attributed to extrinsic factors that allow and promote axonal regeneration in the PNS. Schwann cells (SCs), the PNS myelinating glia, perform major functions in creating a favorable 
environment for axonal growth, stimulating axon outgrowth after injury and rebuilding the myelin sheath of regenerated axons. ${ }^{3-9}$ Therefore, transplantation of SCs has always been considered a feasible method for the repair of nerve injury and functional reconstruction. ${ }^{10-13}$ However, the poor migration of SCs in the astrocyte-rich CNS significantly limits their application in the repair of damaged nerves in the CNS. ${ }^{14-16}$ In addition, astrocytes form a scar barrier to restrict the migration of SCs and do not mix with them. This significantly compromises the ability of SCs to guide regenerating axons into the caudal spinal cord and weakens their ability to mediate remyelination.

To solve this problem, SCs were genetically modified to enhance their ability to break through the scar formed by astrocytes. ${ }^{17}$ In previous studies, the chondroitinase $\mathrm{ABC}$ (ChABC) gene was genetically introduced into SCs to degrade chondroitin sulfate proteoglycans (CSPGs) by cleaving glycosaminoglycan chains, ${ }^{18-20}$ which were responsible for most of the inhibitory effect. ${ }^{19,21}$ However, the direct expression of prokaryotic proteins in mammalian cells is problematic since the newly synthesized chondroitin peptide is secreted through eukaryotic cells. Therefore, the chondroitin enzyme genes were modified to enable the transfected cells to effectively secrete the active chondroitin enzyme, ${ }^{20,22}$ and the full sequence was provided in Supplementary Data. Related research has shown that the sustained expression of modified ChABC by SCs enhanced their ability to traverse the scar formed by astrocytes. ${ }^{23,24}$ Nevertheless, SCs with ChABC did not migrate along the cephalocaudal direction, which significantly limits their effect on nerve repair after central nerve injury.

Based on the combination of molecular biology and nanotechnology, biomaterials with nanoscale features can induce cell migration, adhesion, proliferation, and differentiation. ${ }^{25}$ Superparamagnetic ferric oxide nanoparticles (SPIONs) are considered a highly efficient gene transfection vector ${ }^{26,27}$ and can promote cell migration to the desired direction under the action of MF. In addition, the SPIONs have been certified as a safe magnetic resonance imaging contrast agent by the Food and Drug Administration, which holds many advantages over other gene vectors, such as protection of genes from digestion, low immunogenicity, etc. Following the entry of the target gene binding with SPIONs cells, SPIONs can protect the gene from being degraded by nuclease. Under the action of external $\mathrm{MF}$, the SPIONs/DNA complex rapidly precipitates and contacts with cells, which accelerates the transfection time, and increases the transfection efficiency and DNA endocytosis. ${ }^{28}$
Moreover, polyethyleneimine (PEI) is coated on the surface of SPIONs to produce a cationic surface, which is more favorable for the cell internalization of the carrier. Particularly, an external magnetic field (MF) could produce force along the MF by acting on the internalized SPIONs, promoting cell migration in the desired direction.

In this study, the PEI-SPIONs were firstly used as a gene vector for ChABC to enhance its ability to eliminate CSPGs and promote the crossing of SCs through the barrier of glial scar. In addition, the MF was used to guide cell migration in a specific direction under the force of SPIONs. The purpose of this study was to establish a new strategy for enhancing the fusion of SCs with astrocytes and activate the capability of SCs to support and guide axonal regeneration after spinal cord injury (SCI).

\section{Materials and Methods Synthesis of the PEI-SPIONs/ChABC Complexes and PEI-SPIONs/DNA Complexes}

The PEI-SPIONs ( $1 \mu \mathrm{g} / \mu \mathrm{L})$ (Chemicell, Berlin, Germany) comprised ferrosoferric oxide nanoparticles with branched PEI (25 kDa) as surface modification. The complementary DNA (cDNA) encoding rat ChABC, STK39 (1638 bp, Accession number: NM_013233), was inserted into a pcDNA3.1 transfer plasmid (Genomeditech, Shanghai, China) between its unique SacII and BamHI cutting sites (ChABC plasmid). For comparison, we also synthesized a control pcDNA3.1 transfer plasmid (Genomeditech, Shanghai, China) without the target gene (DNA plasmid). In the presence of different volumes of distilled water, the PEISPIONs and ChABC or DNA were diluted to form different mass ratios of complexes at $\mathrm{pH} 7.4$ and subsequently incubated at room temperature for 30 mins to form complexes.

\section{Characterization of PEI-SPIONs}

Transmission electron microscopy (TEM) (H-600; Hitachi, Tokyo, Japan) was used to analyze the morphology, distribution, and average size of PEI-SPIONs. The zeta potential and size distribution of the PEI-SPIONs were estimated at $20-25^{\circ} \mathrm{C}$ using a zeta potential/nanometer particle size analyzer (DelsaNano; Beckman Coulter, Brea, CA, USA).

\section{Culture and Purification of SCs}

Primary SCs were isolated from sciatic nerves and brachial plexus of Sprague Dawley rats (postnatal days $1-3 ; n=100$, provided by the Laboratory Animal Center of the Fourth 
Military Medical University[FMMU], Xi'an, China) as previously described with some modifications. ${ }^{29,30}$ All experimental procedures were performed in accordance with the Guide for the Care and Use of Laboratory Animals and approved by the Animal Research Committee of The Fourth Military Medical University (Xi'an, China). The epineurium was removed prior to cutting the nerve into small pieces for 2 mins, explants were dissociated with $0.2 \%$ of type II collagenase and $0.25 \%$ trypsin for 26 mins at $37^{\circ} \mathrm{C}$, and mixed evenly every 5 mins. Subsequently, the cells were maintained in Dulbecco's Modified Eagle Medium: Nutrient Mixture F-12 (DMEM/ F12, HyClone, Logan, Utah, USA) containing 15\% fetal bovine serum (FBS; Gibco, Grand Island, State of New York, USA) and 1\% antibiotics (penicillin and streptomycin solution). At $24 \mathrm{hrs}$ after inoculation, the cells were treated with $10 \mu \mathrm{m}$ cytosine cytarabine $\left(10^{-5} \mathrm{M}\right)$ for $48 \mathrm{hrs}$ to inhibit the proliferation of fibroblasts. SCs were maintained in DMEM/F12 (HyClone) containing 15\% FBS (Gibco), 1\% antibiotics (penicillin and streptomycin solution) supplemented with $2 \mathrm{mM}$ forskolin (Sigma-Aldrich, St. Louis, MO, USA) and $20 \mathrm{mg} / \mathrm{mL}$ bovine pituitary extract (BPE; Biomedical Technologies Inc., Stoughton, MA, USA). These cultures were highly enriched in SCs $(>95 \%)$ based on the immunostaining of the SC marker $\mathrm{S} 100$ and p75 neurotrophin receptor (Figure 1A-D).

\section{Culture and Purification of Astrocytes}

Primary astrocytes were prepared from the cerebral cortices of Sprague Dawley rats (postnatal days $1-3 ; n=50$, provided by the Laboratory Animal Center of the FMMU) as previously described with some modifications. ${ }^{30}$ In brief, the brain is carefully extracted under a dissecting microscope in Hank's balanced salt solution. Subsequently, the meninges were removed and the brain was cut into small pieces, digested in $0.25 \%$ trypsin and $0.2 \%$ type IV collagenase for 30 mins at $37^{\circ} \mathrm{C}$, and mixed evenly every 5 mins. The cells were plated on T75 flasks coated with poly-L-lysine (PLL) and incubated in DMEM/ F12 (HyClone) containing 10\% FBS (Gibco) and 1\% antibiotics (penicillin and streptomycin solution). The primary cultured astrocytes were shaken for $24 \mathrm{hrs}$ (200 rpm) at $37^{\circ} \mathrm{C}$, and microglia and oligodendrocytes were removed in the process. The astrocytes were washed twice with phosphate-buffered saline (PBS; HyClone) solution to remove any suspended cells. Finally, the medium was replaced with fresh DMEM/F12 (HyClone) containing $10 \%$ FBS and $1 \%$ antibiotics (penicillin and streptomycin solution). These cultures were highly enriched in astrocytes ( $>90 \%$ ) based on the immunostaining of the astrocyte marker glial fibrillary acidic protein (GFAP) (Figure 1E-G).

\section{Magnetofection of SCs}

A schematic diagram of the magnetofection is shown in Figure $1 \mathrm{H}$. The plasmid was diluted to a concentration of $0.08 \mu \mathrm{g} / \mu \mathrm{L}$ with distilled water. The weight ratio of the PEISPIONs to the ChABC plasmids was $1: 4 ; 4 \mu \mathrm{L}$ of PEISPIONs $(1 \mu \mathrm{g} / \mu \mathrm{L})$ solution was added to $200 \mu \mathrm{L}$ of diluted plasmid solution and mixed immediately through vigorous pipetting. ${ }^{27}$ The mixture was prepared at room temperature for 30 mins by combining cDNA with magnetic nanoparticles to form PEI-SPIONs/cDNA complexes.

Primary SCs were cultured in a six-well plate with DMEM/F12 (HyClone) containing 15\% FBS, 1\% antibiotics (penicillin and streptomycin solution) supplemented with $2 \mathrm{mM}$ forskolin (Sigma-Aldrich) and $20 \mathrm{mg} / \mathrm{mL} \mathrm{BPE}$ (Biomedical Technologies Inc.), and cultured to $70-80 \%$ of confluency. Cells were washed twice with sterile PBS (HyClone) or DMEM/F12 (HyClone) without serum prior to transfection, and the medium was replaced with $1.8 \mathrm{~mL}$ fresh serum-free medium per well. Subsequently, $200 \mu \mathrm{L}$ of the prepared PEI-SPIONs/ChABC complex or PEISPIONs/ChABC complex solution was added to each well of the six-well cell culture plate. After mixing, the six-well cell culture plate was placed on top of a commercial magnetic neodymium-iron-boron multiwell plate (MagnetoFACTOR-96 plate; Chemicell), the strength of which was $0.3 \mathrm{~T}$, for $4 \mathrm{hrs}$ of incubation at $37^{\circ} \mathrm{C}$ in an atmosphere containing $5 \%$ carbon dioxide. The special geometry of the MagnetoFACTOR-96 plate, the polarity of which is alternated north and south, ensures that the strength of the MF in each well is uniform and equal.

After magnetofection, the medium was replaced with fresh serum containing 15\% FBS, 1\% antibiotics (penicillin and streptomycin solution) supplemented with $2 \mathrm{mM}$ forskolin (Sigma-Aldrich) and $20 \mathrm{mg} / \mathrm{mL}$ BPE (Biomedical Technologies Inc.), and the magnetofected SCs with PEISPIONs/ChABC complexes (PEI-SPIONs/ChABC/SCs) or magnetofected SCs with PEI-SPIONs/DNA complexes (PEI-SPIONs/DNA/SCs) were cultured for further use.

\section{Cell Counting Kit 8 (CCL-8) Assays}

The viability and cytotoxicity of cells were analyzed using the CCK-8 assay (Dojindo, Kumamoto, Japan) according 

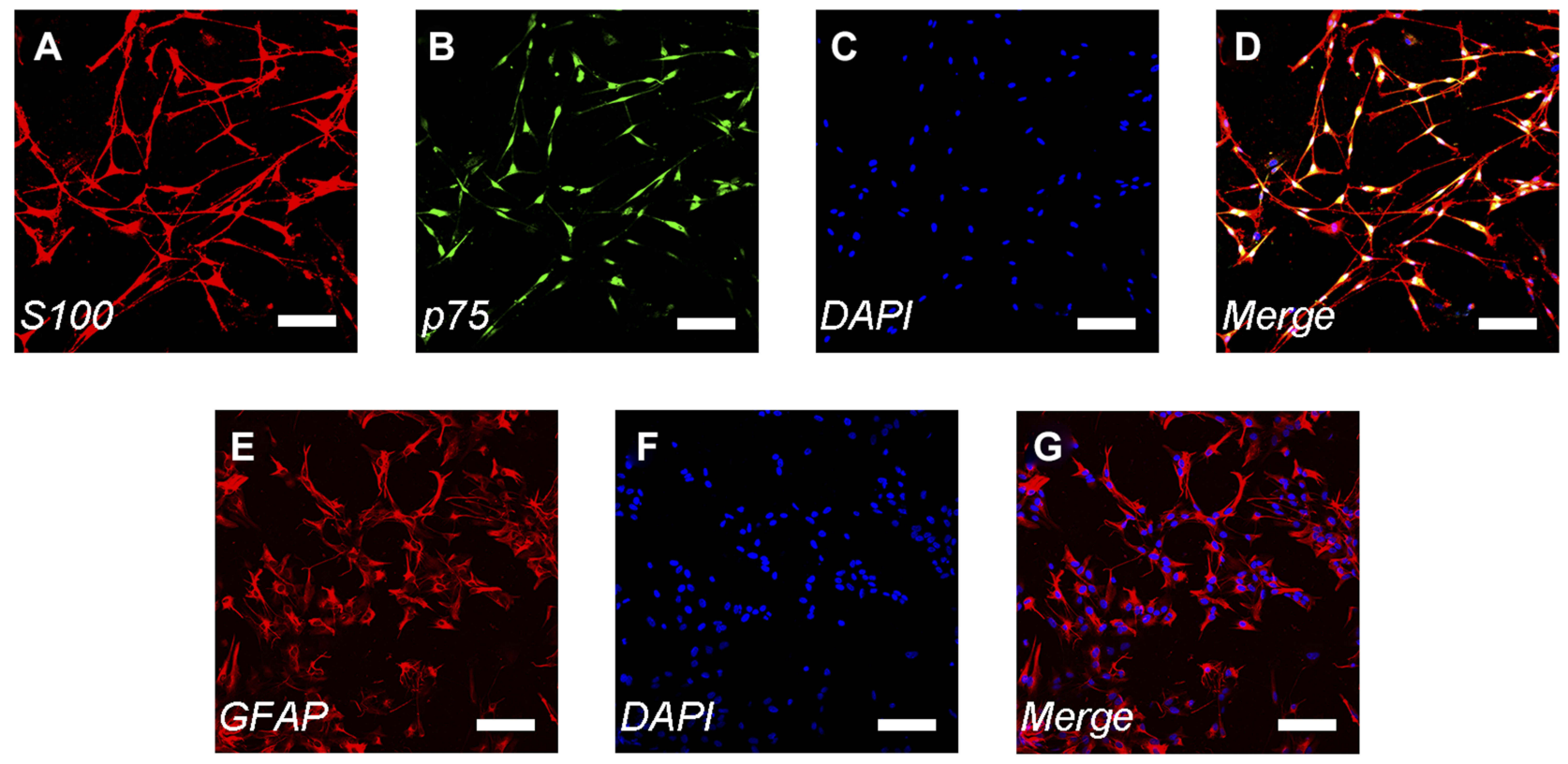

H

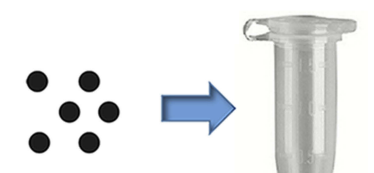

SPIONS
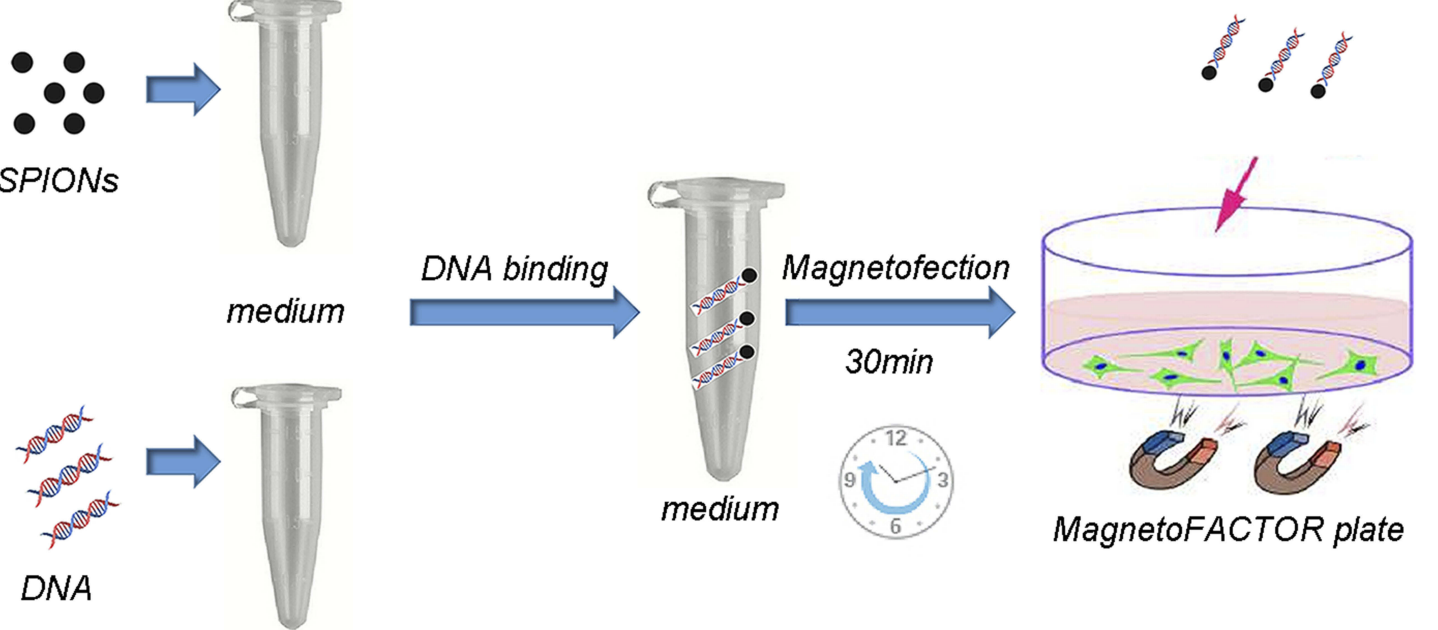

Purified SCs

SPIONs

XDDOD DNA

Figure I Immunofluorescence staining of Schwann cells/astrocytes and schematic diagram of the magnetofection.

Notes: SC primary cultures stained for SI00 (A), p75 (B), nuclei with DAPI (C), and merge file showed a purity of $>95 \%$ SCs (D). Astrocyte primary cultures stained for GFAP (E), nuclei with DAPI (F) and merge file showed a purity of $>90 \%$ astrocytes (G). Flowing chart of magnetofection (H). Scale bar (A-G), I00 $\mu$ m.

Abbreviations: SCs, Schwann cells; DAPI, 4',6-diamidino-2-phenylindole; SPIONs, superparamagnetic iron oxide nanoparticles

to the instructions provided by the manufacturer. Briefly, different concentrations of PEI-SPIONs $(0,1,2,4$, and $8 \mu \mathrm{g} / \mathrm{mL}$ ) were magnetofected into $\mathrm{SC}$ in 96 -well plates. After 24 or $72 \mathrm{hrs}$ of incubation, the SCs were washed 3 times with sterile PBS (HyClone); subsequently, $100 \mu \mathrm{L}$ of fresh medium containing $10 \mu \mathrm{L}$ of CCK-8 reagent was added to each well and incubated for $4 \mathrm{hrs}$ at $37^{\circ} \mathrm{C}$ under a humidified atmosphere with $5 \%$ carbon dioxide. The absorbance was measured at $450 \mathrm{~nm}$ using a microplate reader (Synergy H1, BioTek, Vermont, USA).

\section{LIVE/DEAD Assay}

LIVE/DEAD assays were performed using a LIVE/DEAD Cell Imaging Kit (Thermo Fisher Scientific Inc., Wyman Street, Waltham, MA, USA) in a 24-well plate according to the instructions provided by the manufacturer. Live and 
dead cells became visible with green and red color. Briefly, different concentrations of PEI-SPIONs $(0,1,2,4$, and $8 \mu \mathrm{g} / \mathrm{mL}$ ) were magnetofected into SCs. After $24 \mathrm{hrs}$ or $72 \mathrm{hrs}$ of incubation, the cells were washed thrice with PBS (HyClone), followed by the addition of the staining solution. Cell viability was analyzed by counting the live and dead cells after incubation for $15 \mathrm{mins}$ at $37^{\circ} \mathrm{C}$. The number of viable (green) or dead (red) cells was counted using the Image Pro Plus software (Media Cybernetics, Bethesda, MI, USA).

\section{Electron Microscopic Analysis of SCs After Magnetofection with PEI-SPIONs}

Primary SCs were plated on a slide coated with PLL (SigmaAldrich) and magnetofected with PEI-SPIONs $(2 \mu \mathrm{g} / \mathrm{mL})$ to study their morphology after magnetofection. At $6 \mathrm{hrs}$ after magnetofection, the cells were washed with sterile PBS (HyClone) to remove cells that had fallen off or died, followed by fixation and dehydration with serial ethanol solution. Subsequently, the cells were dried at room temperature in a vacuum atmosphere and sputter-coated with gold; and the samples were observed using a scanning electron microscope (S-3400N; Hitachi).

Primary SCs were seeded in two wells in the six-well plate and treated with PEI-SPIONs $(2 \mu \mathrm{g} / \mathrm{mL})$ to observe the presence of PEI-SPIONs in SCs. After $24 \mathrm{hrs}$ of cultivation, the cells were handled according to the standard protocol for TEM imaging (H-600; Hitachi).

\section{Gene Expression Analysis Through Quantitative Real-Time Polymerase Chain Reaction (qRT-PCR)}

Total RNA from SCs magnetofected with different safety concentrations of PEI-SPIONs ( 1 and $2 \mu \mathrm{g} / \mathrm{mL}$ ) after $72 \mathrm{hrs}$ was extracted using the TRIzol reagent (Invitrogen, Carlsbad, CA, USA) according to the protocol provided by the manufacturer. Subsequently, total RNA was isolated and normalized to the number of cells. cDNA was synthesized using PrimeScript ${ }^{\mathrm{TM}}$ RT Master Mix (TaKaRa, Dalian, Liaoning Province, China), and qRT-PCR was performed using a FastStart Essential DNA Green Master (Roche, Basel, Switzerland) to measure the expression of ChABC.

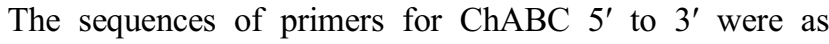
follows: upper, AGACCCGATGGAACTGCTTG; lower, CTTTCGCCCACTGAAAACGG. The qRT-PCR parameters used were: denaturation at $95^{\circ} \mathrm{C}$ for $30 \mathrm{~s}$; primer annealing at $59^{\circ} \mathrm{C}$ for $30 \mathrm{~s}$; and elongation products at $72^{\circ} \mathrm{C}$ for $40 \mathrm{~s}$. All data were calculated using the $2^{-\Delta \Delta \mathrm{Ct}}$ method. The housekeeping gene $\beta$-actin was used to normalize the levels of mRNA. The sequences of primers for $\beta$-actin $5^{\prime}$ to $3^{\prime}$ were as follows: upper, AGATCCTGACCGAGCGTGGC; lower, CCAGGGAGGAAGAGGATGCG. Assays were performed in triplicate.

\section{Western Blotting Analysis of ChABC}

Western blotting was performed as previously described. ${ }^{31}$ At $72 \mathrm{hrs}$, after removal of the media, SCs or SCs magnetofected with PEI-SPIONs/DNA or PEI-SPIONs/ChABC were seeded in six-well plates, washed twice with PBS (HyClone), and lysed using radioimmunoprecipitation assay buffer $(50 \mathrm{mM}$ Tris pH7.4, $150 \mathrm{mM}$ sodium chloride $[\mathrm{NaCl}], 1 \%$ Triton $\mathrm{X}-100,1 \% \mathrm{mM}$ sodium deoxycholate, $0.1 \%$ sodium dodecyl sulfate, ethylenediaminetetraacetic acid, sodium orthovanadate, sodium fluoride, leupeptin) containing $1 \mathrm{mM}$ phenylmethanesulfonyl fluoride. The lysates were collected from the plates through scraping and subjected to centrifugation at $10,000 \times \mathrm{g}$ for $5 \mathrm{mins}$ at $4^{\circ} \mathrm{C}$. Total protein concentration was quantified using the bicinchoninic acid (Beyotime Institute of Biotechnology, Shanghai, China) assay. Briefly, $30 \mu \mathrm{L}$ of total proteins was separately electrophoresed in 10\% sodium dodecyl sulfate-polyacrylamide gel electrophoresis acid gel and transferred onto a polyvinylidene difluoride membrane at 0.8 $\mathrm{mA} / \mathrm{cm}^{2}$ for $2 \mathrm{hrs}$. After blocking with a blocking solution (5\% skimmed milk in Tris-buffered solution plus Tween-20: $50 \mathrm{mM}$ Tris-hydrochloride, $150 \mathrm{mM} \mathrm{NaCl}, \mathrm{pH}=7.5,0.1 \%$ volume/volume Tween 20) at room temperature for $2 \mathrm{hrs}$, the membranes were incubated at $4{ }^{\circ} \mathrm{C}$ overnight with mouse antiChABC monoclonal antibody at 1:1,000 (Novus Biologicals, $\mathrm{CO}$, USA) and rabbit anti- $\beta$-tubulin monoclonal antibody at 1:3000 (Cat. 2128; CST, Boston, MA, USA). After washing twice in Tris-buffered solution plus Tween-20 for 10 mins, the membranes were incubated with a horseradish peroxidaseconjugated secondary antibody. This was followed by washing thrice with PBS (HyClone) for 5 mins. The membranes were examined for immunoreactive proteins using Clarity ${ }^{\mathrm{TM}}$ Western ECL Substrate (BIO-RAD, CA, USA) and analyzed via the GE AI600 system (General Electric Company, Boston, MA, USA). $\beta$-tubulin was used as an internal control.

\section{Preparation of Animals and Tissues}

All animal procedures were conducted using a protocol reviewed and approved by the Institutional Ethics Committee of the FMMU. Male Sprague Dawley rats $(n=6$, 25-40 g, 2-3 weeks old), provided by the Laboratory Animal Center of the FMMU, were anesthetized through an 
intraperitoneal injection of $1 \%$ sodium pentobarbital solution (40 mg/kg) and transcardially perfused with PBS (HyClone) and $500 \mathrm{~mL}$ of $4 \%$ paraformaldehyde in $\operatorname{PBS}(0.1 \mathrm{M}$, $\mathrm{pH}=7.4)$. The spinal cord was harvested at the thoracic level and specimens were immersed in $4 \%$ paraformaldehyde for $4 \mathrm{hrs}$ at $4^{\circ} \mathrm{C}$. Subsequently, the cord was sequentially immersed in $25 \%$ glucose in PBS (HyClone) at $4^{\circ} \mathrm{C}$ until the tissue sank. Afterward, 8- $\mu \mathrm{m}$ serial sagittal sections were cut using a cryostat (CM1850; LEICA, Wetzlar, Hesse, Germany).

\section{ChABC Activity in vitro}

The dimethylmethylene blue (DMB) (Sigma-Aldrich) Assay was used to measure the level of sulfated glycosaminoglycan after degradation of chondroitin sulfate by $\mathrm{ChABC}$ or medium to evaluate the enzymatic activity of $\mathrm{ChABC}$ in SCconditioned medium. ${ }^{32}$ For the DMB assay, $10 \mu \mathrm{L}$ of the medium was collected daily from SCs, PEI-SPIONs /ChABC/SCs, or pure ChABC cultures incubated with 10 $\mu \mathrm{L}$ of chondroitin sulfate $(0.25 \mathrm{mg} / \mathrm{mL})$ for $2 \mathrm{hrs}$ at $37^{\circ} \mathrm{C}$ as described previously. ${ }^{32,33}$ DMB solution $180 \mu \mathrm{L}$ (16 mg of DMB, $2.37 \mathrm{~g}$ of NaCl, $3.04 \mathrm{~g}$ of glycine, $75 \mathrm{~mL}$ of $0.1 \mathrm{M} \mathrm{HCl}$ in $1 \mathrm{~L}$ of $\mathrm{H}_{2} \mathrm{O}$ ) was mixed with $20 \mu \mathrm{L}$ of the aforementioned reaction sample for $15 \mathrm{mins}$ at $37^{\circ} \mathrm{C}$ and measured using a microplate reader (Synergy H1; BioTek) at $660 \mathrm{~nm}$. The control group represented the absorbance of the chondroitin sulfate with the DMEM/F12 (HyClone). A linear standard curve, generated from purified $\mathrm{ChABC}$, was used to evaluate the enzymatic activity of ChABC.

Cryostat sections were incubated in PBS (HyClone), in conditioned medium collected from PEI-SPIONs/ChABC/ SCs cultures, or in PBS (HyClone) containing ChABC (300 $\mathrm{mU} / \mathrm{mL}$ ) for $3 \mathrm{hrs}$ at $37^{\circ} \mathrm{C}$ to confirm the bioactivity of ChABC to degrade CSPGs in the spinal cord tissue. ${ }^{33,34}$ Moreover, the ability of astrocytes to express CSPGs in vitro was also demonstrated. Immunostaining of the incubated sections or astrocytes for CS-56 and GFAP was performed as described in the immunofluorescence staining subsection below.

\section{Inverted Coverslip Migration Assays}

The inverted coverslip assays were performed as previously described. $^{35,36}$ The SCs, PEI-SPIONs/DNA/SCs, or PEISPIONs/ChABC/SCs were trypsinized with $0.125 \%$ trypsin and resuspended at a density of $2 \times 10^{6}$ cells $/ \mathrm{mL}$. Briefly, these three kinds of cells were plated on coverslip fragments $\left(\sim 5 \mathrm{~mm}^{2}\right)$ coated with PLL. The fragments were cultured for 24-36 hrs until SCs adhered to the surface. Subsequently, the coverslips were washed thrice with sterile PBS (HyClone) to remove nonadherent cells and inverted (ie, cells facing downward) onto a glass-bottom cell culture dish coated with astrocytes at a density of $1 \times 10^{5}$ for 2 days to allow cell migration in DMEM/F12 medium containing 15\% FBS, $1 \%$ antibiotic (penicillin and streptomycin solution) supplemented with $2 \mathrm{mM}$ forskolin (Sigma-Aldrich) and $20 \mathrm{mg} / \mathrm{mL}$ BPE (Biomedical Technologies Inc.). An external static magnet (cube magnet; grade; cube side length: $50 \mathrm{~mm}$; MF strength: 1.4 tons) was placed in parallel on the edge of the coverslip for further analysis. Subsequently, the cells were fixed and immunofluorescence staining was performed. The distance of cell migration away from the edge of the fragment was analyzed. The maximum distance of migrated SCs to the edge of the marker was measured and recorded, and the number of SCs that migrated at a distance of approximately $1800 \mu \mathrm{m}$ was calculated. In addition, the angle $\theta$ between the long axis of each migrated SC and the outward MF direction was also measured. The orientation index, which represented orientation of SC migration, defined as $\mathrm{Oi}=\cos (\theta)$, with the angle $0, \theta, \pi / 2(\mathrm{Oi}=1$ when the direction of $\mathrm{SC}$ migration overlaps with the $\mathrm{MF}$ direction, $\mathrm{Oi}=0.5$ or 0 when the angle is $60^{\circ}$ or $90^{\circ}$ between the direction of SC migration and the MF direction). Values of Oi closer to 1 indicate a migration angle closer to the direction of the MF. In each experiment, more than six regions were randomly selected, and the average measured number of SCs in each region was 30. All migrations were performed under conditions of aphidicolin (1.5 $\mu \mathrm{g} / \mathrm{mL}$ ) inhibition to determine that movement away from the substance was not the result of proliferation.

\section{SC and Astrocyte Confrontation Assays}

$\mathrm{SC}$ and astrocyte confrontation assays were performed as previously described with some modification. ${ }^{35,37}$ Briefly, $25 \mu \mathrm{L}$ strips of astrocytes (density: $6 \times 10^{5} / \mathrm{mL}$ ) or SCs (density: $2 \times 10^{6} / \mathrm{mL}$ ) were plated parallel to each other on a PLL-coated confocal dish. After attachment for $3 \mathrm{hrs}$, the cells were washed with sterile PBS (HyClone). The MF was applied in parallel to the SC strips on the astrocyte side. The cells were cultured for 7 days using $15 \%$ DMEM/F12 medium containing 15\% FBS, 1\% antibiotic (penicillin and streptomycin solution) supplemented with $2 \mathrm{mM}$ forskolin (Sigma-Aldrich) and $20 \mathrm{mg} / \mathrm{mL} \mathrm{BPE}$ (Biomedical Technologies Inc.) and migrated toward each other. Subsequently, the cells were fixed and immunofluorescence was performed using anti-GFAP for astrocytes and anti-S100 for SCs as described in the following subsection. SCs that crossed the cell boundary and 
migrated to the astrocyte region were counted and more than six areas were randomly selected per condition.

\section{Immunofluorescence Staining}

Immunofluorescence staining was performed as previously described with some modification. ${ }^{38}$ The cells were fixed using 4\% paraformaldehyde for $15 \mathrm{mins}$ at room temperature, rinsed with PBS (HyClone) for 5 mins thrice, blocked in $0.2 \%$ Triton X-100, washed as above, and incubated with $10 \%$ normal goat serum for $1 \mathrm{hr}$. Subsequently, the cells were incubated with primary antibodies overnight at $4^{\circ} \mathrm{C}$. The antibodies used were as follows: polyclonal anti-S100 protein antibody (1:100; Abcam, Cambridge, UK), polyclonal mouse anti-p75 antibody (1:100; Abcam, Cambridge, UK), polyclonal chicken anti-GFAP antibody (1:1,000; Abcam), and monoclonal mouse anti-CS56 (1:200; Sigma-Aldrich). After rewarming for $1 \mathrm{hr}$ at room temperature, the cells were incubated with indocarbocyanine-conjugated goat anti-chicken (1:500; Beijing ComWin Biotech Co., Ltd., Beijing, China), indocarbocyanineconjugated goat anti-mouse (1:200; Beijing ComWin Biotech Co., Ltd.), or fluorescein-conjugated goat antirabbit (1:50; Beijing ComWin Biotech Co., Ltd.) secondary antibodies for $1 \mathrm{hr}$ at $37^{\circ} \mathrm{C}$. After washing with thrice, the cells were stained with 4',6-diamidino-2-phenylindole solution (Sigma-Aldrich) for 5 mins at room temperature. SCs were identified using primary anti-p75 and anti-S100 antibodies, while astrocytes were identified using anti-GFAP antibodies. The results of immunofluorescence staining were analyzed using confocal microscopy (A1+, Nikon, Tokyo, Honshu Island, Japan).

Initially, sections of spinal cord were washed in PBS (HyClone) for 5 mins and incubated in a blocking solution of $10 \%$ normal goat serum with $0.2 \%$ Triton X-100 for $1 \mathrm{hr}$ at room temperature. The sections were incubated with antibodies against polyclonal chicken anti-GFAP (1:1000) or monoclonal mouse anti-CS56 (1:500) diluted in Immunol Staining Primary Antibody Dilution Buffer (Beyotime, Institute of Biotechnology) overnight. After rewarming for $1 \mathrm{hr}$ at room temperature, the sections were rinsed thrice with PBS (HyClone, Utah, USA) and incubated with secondary antibodies: indocarbocyanine-conjugated goat anti-chicken (1:500; Beijing ComWin Biotech Co., Ltd.) or indocarbocyanine-conjugated goat anti-mouse (1:200; Beijing ComWin Biotech Co., Ltd.) for $1 \mathrm{hr}$ at $37^{\circ} \mathrm{C}$. Sections were rinsed thrice with PBS (HyClone) and covered with a square cover slide. The results of immunofluorescence staining were analyzed through confocal microscopy (A1+, Nikon).

\section{Statistical Analysis}

All data in this study conformed to the positive distribution and expressed as the mean \pm standard deviation. Multiple comparisons of groups were conducted using one-way analysis of variance with the SPSS version 20 software package (IBM Corporation, Armonk, NY, USA). The values of Oi were expressed as their respective medians and interquartile ranges. For the DMB assay in vitro, significant differences between the groups were assessed using the parametric Student's $t$-test. Differences with $\mathrm{p}<0.05$ denoted statistical significance.

\section{Results}

\section{Characterizations of the PEI-SPIONs and PEI-SPIONs/ChABC Complexes}

TEM was used to analyze the morphological appearance of PEI-SPIONs, the average diameter of PEI-SPIONs was 27.1 $\pm 2.6 \mathrm{~nm}$ (Figure 2A). The saturation magnetization at 3.382 Oe was $61.62 \mathrm{emu} / \mathrm{g}$ (Figure 2B). The particle size distribution and zeta potentials of the PEI-SPIONs and PEI-SPIONs $/ \mathrm{ChABC}$ complexes were analyzed using Brownian motion video analysis laser scattering microscopy to evaluate the plasmid-binding ability of PEI-SPIONs. The average diameter and positive charge of PEI-SPIONs were $108.2 \pm 5.1 \mathrm{~nm}$ (Figure 2C) and $48.4 \pm 1.8 \mathrm{mV}$ (Figure 2D), respectively. In contrast, the PEI-SPIONs/ChABC had an average diameter of $122.7 \pm 1.4 \mathrm{~nm}$ (Figure 2E) and a positive charge of 39.7 \pm 2.5 $\mathrm{mV}$ (Figure 2F). These data indicate that PEI-SPIONs have positive charges after binding to the plasmid, which is suitable for gene transfection into cells.

\section{Magnetofection of SCs}

In our previous study, we found that the presence of PEISPIONs could be detected on the cell membrane surface 2 hrs after magnetofection. The transfer of particles to the cytoplasm was initiated after $6 \mathrm{~h}$; particles were detected in the cytoplasm 12-18 hrs after transfection. In the present study, particles were observed on the surface of SCs after $6 \mathrm{hrs}$ of magnetofection (PEI-SPIONs, $2 \mu \mathrm{g} / \mathrm{mL}$ ); however, there were no particles observed in the normal control SCs (Figure 3A-C). At 24 hrs after magnetofection, a cluster of PEI particles was found within the SCs (Figure 3D-F), indicating that PEI-SPIONs attached to the cells and were transferred into the cytosol at the corresponding timepoints. 
A

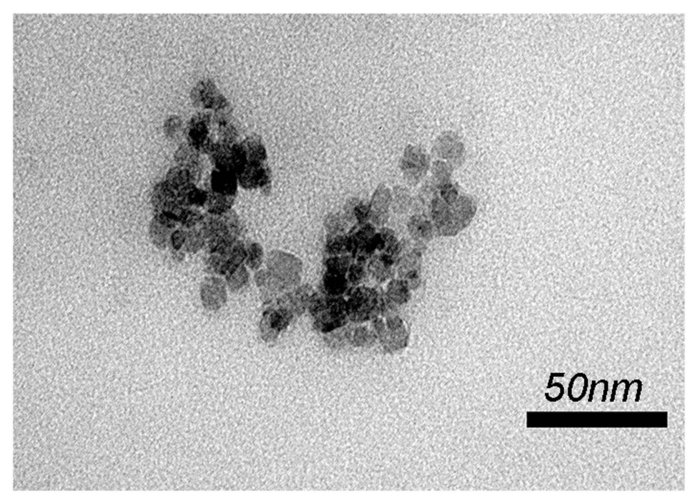

C

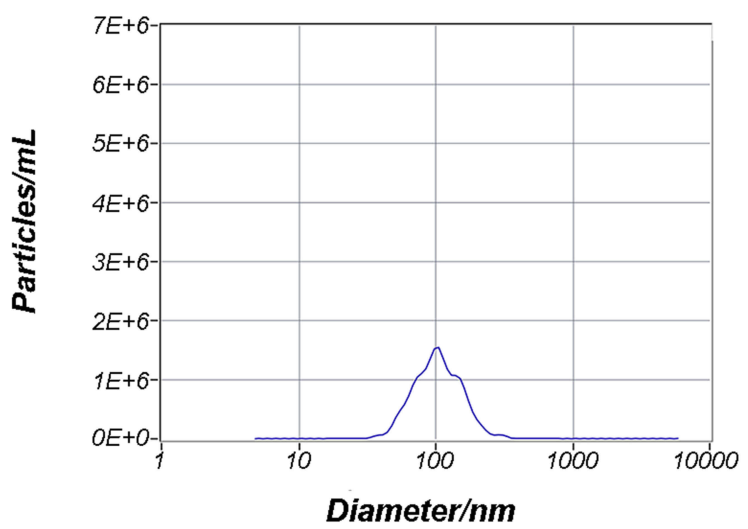

E

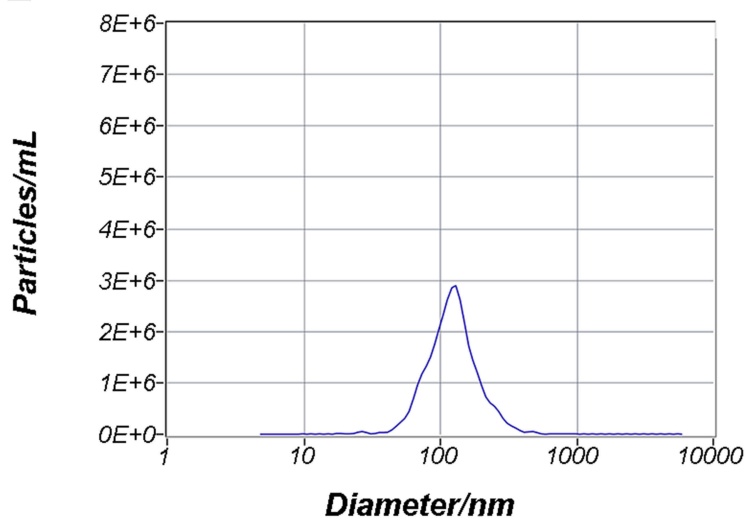

B

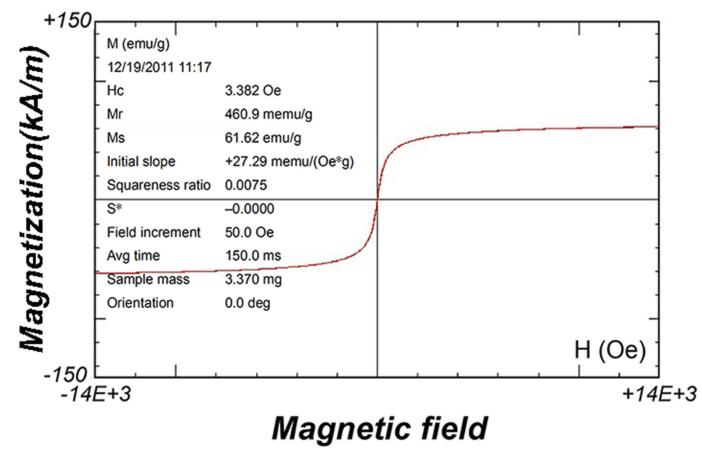

D

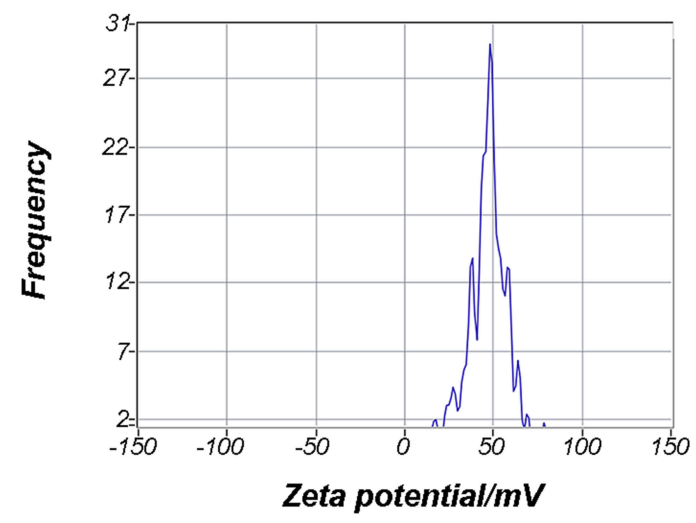

F

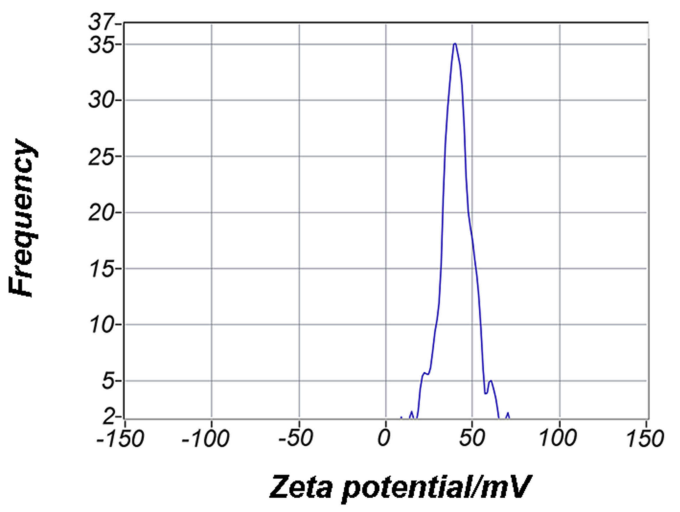

Figure 2 Characterization of PEI-SPIONs.

Notes: (A) TEM analysis of PEI-SPIONs. (B) Magnetization parameters of PEI-SPIONs. (C) Overall distribution of PEI-SPIONs sizes. (D) Zeta potential of PEI-SPIONs. (E) Overall distribution of PEI-SPIONs/ChABC complex sizes. (F) Zeta potential of the PEI-SPIONs/ChABC complex.

Abbreviations: avg, average; PEI-SPIONs, polyethylenimine-coated superparamagnetic iron oxide nanoparticles; TEM, transmission electron microscopy

\section{Cytotoxicity Analysis of PEI-SPIONs}

The CCK-8 assay was performed at $24 \mathrm{hrs}$ and $72 \mathrm{hrs}$ after magnetofection to evaluate the cytotoxicity of PEI-SPIONs. We performed the LIVE/DEAD assay to further investigate the cytotoxicity of PEI-SPIONs. At 24 hrs after magnetofection, the percentage of dead cells at a PEI-SPIONs of $0-4 \mu \mathrm{g} / \mathrm{mL}$ was $3.2-4.8 \%$. When the concentration was increased to $8 \mu \mathrm{g} /$ $\mathrm{mL}$, the percentage of dead cells also increased to $15.83 \%$ $\pm 1.46 \%$ (Figure $4 \mathrm{~A}-\mathrm{E}$ and $\mathrm{K}$ ). At $72 \mathrm{hrs}$ after magnetofection, the percentage of dead cells at a PEI-SPION concentration of $0-2 \mu \mathrm{g} / \mathrm{mL}$ was $3.7-6.3 \%$. When the concentration was increased to $4 \mu \mathrm{g} / \mathrm{mL}$ and $8 \mu \mathrm{g} / \mathrm{mL}$, the percentage of dead 
cells also increased to $13.07 \% \pm 0.97 \%$ and $17.73 \% \pm 0.83 \%$, respectively (Figure 4F-J and K). At 24 hrs, PEI-SPIONs did not have a significant effect on cell viability at concentrations of $0,1,2$, and $4 \mu \mathrm{g} / \mathrm{mL}$. However, when the concentration of PEI-SPIONs was increased to $8 \mu \mathrm{g} / \mathrm{mL}$, the viability of SCs after magnectofection was significantly reduced by $38.2 \%$. At 72 hrs, PEI-SPIONs did not show a significant effect on cell viability at concentrations of 0,1 , and $2 \mu \mathrm{g} / \mathrm{mL}$. Nevertheless, when the concentration of PEI-SPIONs was increased to $4 \mu \mathrm{g} / \mathrm{mL}$ and $8 \mu \mathrm{g} / \mathrm{mL}$, the viability of SCs was reduced by $21.3 \%$ and $44.7 \%$, respectively (Figure $4 \mathrm{~L}$ ). These results indicate that a PEI-SPION concentration of $<4 \mu \mathrm{g} / \mathrm{mL}$ is a safe dose. Therefore, in the remaining experiments of this study, the transfection was performed at a concentration of $2 \mu \mathrm{g} / \mathrm{mL}$.

\section{Enhanced Expression of ChABC in PEI-SPIONs/ChABC/SCs}

The concentration and weight ratio of PEI-SPIONs in PEISPIONs/ChABC plasmids exerted effects on the expression of $\mathrm{ChABC}$ in PEI-SPIONs/ChABC/SCs. At safe doses of PEI-SPIONs (ie, concentration of $1 \mu \mathrm{g} / \mathrm{mL}$ and $2 \mu \mathrm{g} / \mathrm{mL}$ ), the mRNA level was higher at a weight ratio of PEISPIONs to plasmids of $1: 4$ than $1: 2$. With the increase in the weight ratio of PEI-SPIONs/ChABC plasmids from 1:2 to $1: 4$, the mRNA levels of ChABC increased by 30 -and 18.7 -fold at $1 \mu \mathrm{g} / \mathrm{mL}$ and $2 \mu \mathrm{g} / \mathrm{mL}$ of PEI-SPIONs, respectively. Notably, the mRNA levels of PEI-SPIONs/ChABC containing $2 \mu \mathrm{g} / \mathrm{mL}$ PEI-SPIONs with a weight ratio of 1:4 of ChABC plasmids were 232.4-fold higher than those detected in the normal control SCs (Figure 5A). In addition, the protein levels of $\mathrm{ChABC}$ in normal control SCs, control PEI-SPIONs/DNA/SCs $(2 \mu \mathrm{g} / \mathrm{mL}$, ratio of $1: 4)$, and PEISPIONs/ChABC/SCs $(2 \mu \mathrm{g} / \mathrm{mL}$, ratio of $1: 4)$ were examined through Western blotting. The ChABC protein was highly expressed in PEI-SPIONs/ChABC, whereas ChABC protein was not detected in normal control SCs and PEI-SPIONs/DNA/SCs (2 $\mu \mathrm{g} / \mathrm{mL}$, ratio of $1: 4)$ (Figure 5B). These results indicated enhanced expression of ChABC in ChABC-magnetofected SCs.
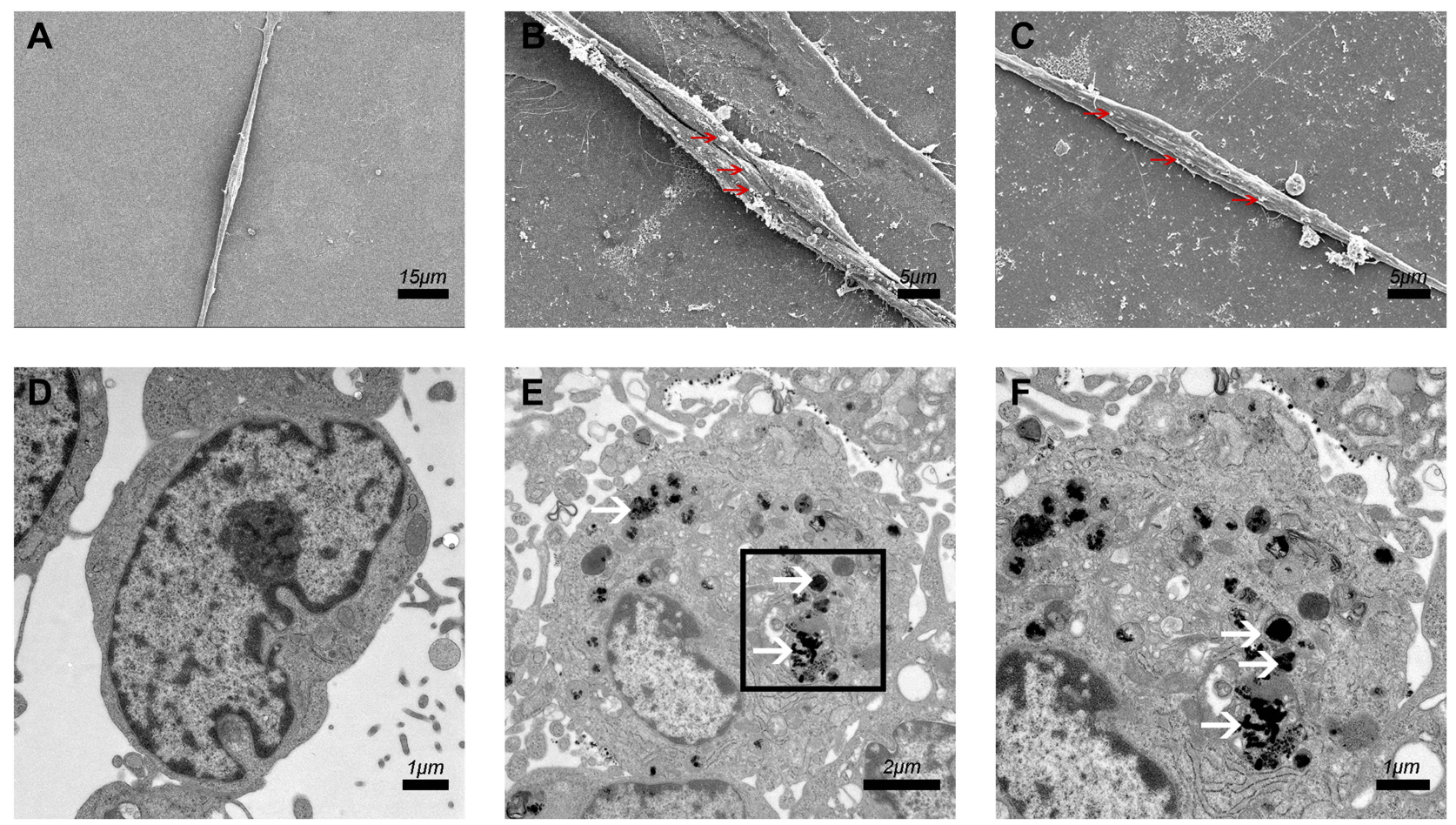

Figure 3 Cellular internalization of PEI-SPIONs.

Notes: (A) SEM of SCs in the normal control group; no relevant particles were detected on the surface of the cells. (B, C) At 6 hrs after magnetofection (PEI-SPIONs, $2 \mu \mathrm{g} /$ $\mathrm{mL}$ ); red arrows indicate PEI-SPIONs on the surface of cells. (D) No PEI-SPIONs in the cytoplasm of normal control SCs detected through TEM. (E, F) At 24 hrs after magnetofection (PEI-SPIONs, $2 \mu \mathrm{g} / \mathrm{mL}$ ); white arrows indicate the internalized PEI-SPIONs in the cytoplasm. (F) Higher magnification of the boxed area in (E). Abbreviations: PEI-SPIONs, polyethylenimine-coated superparamagnetic iron oxide nanoparticles; SCs, Schwann cells; SEM, scanning electron microscopy; TEM, transmission electron microscopy. 

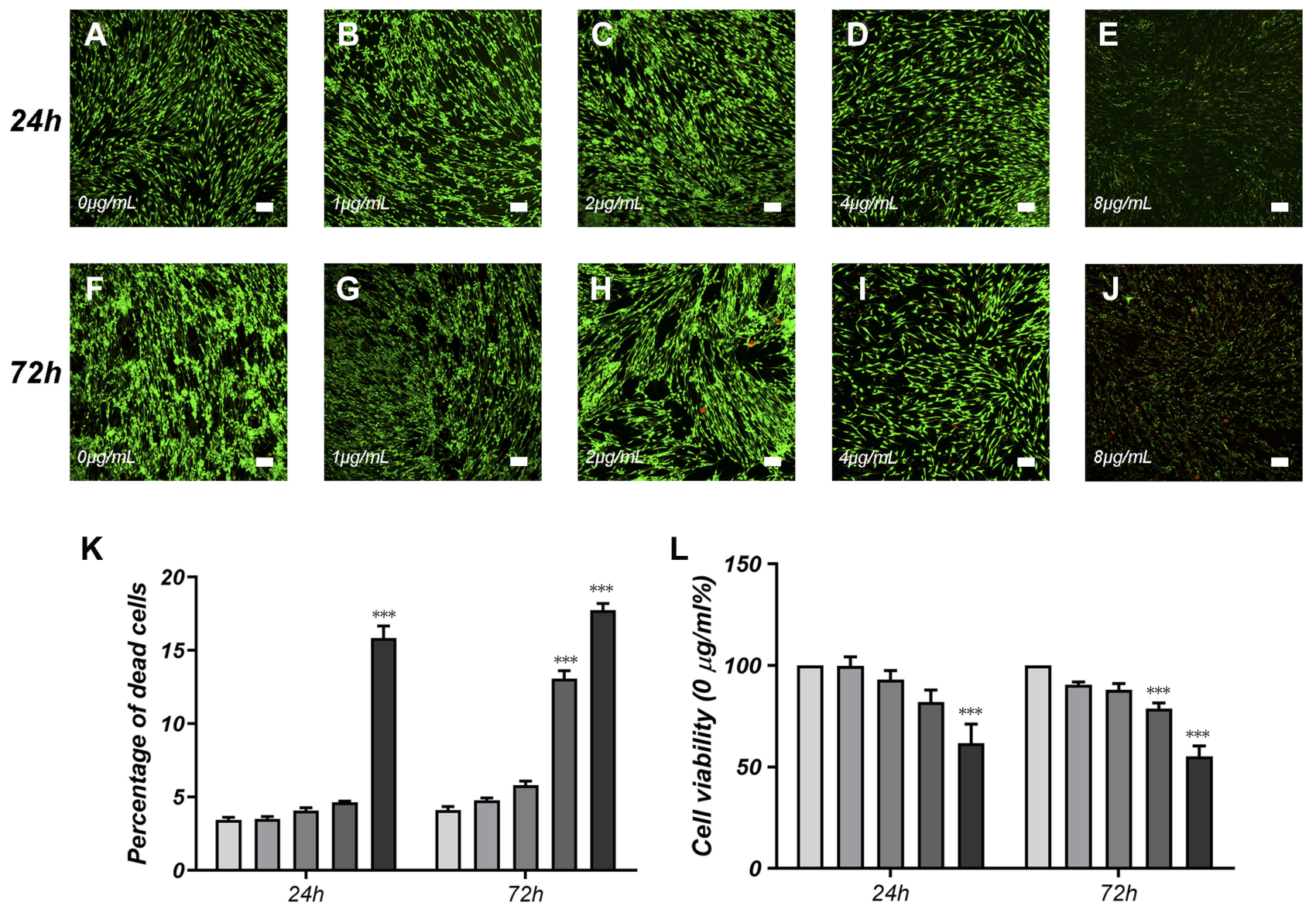

Incubation time

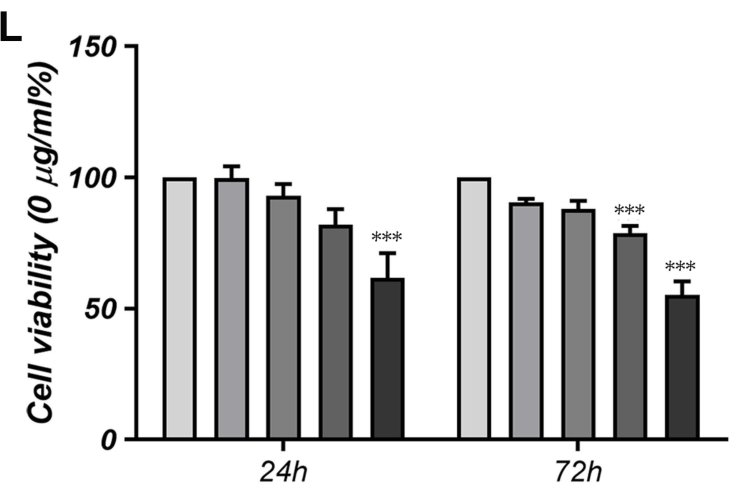

Incubation time

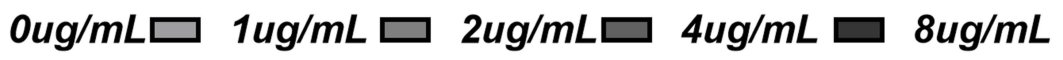

Figure 4 Cytotoxicity tests of PEI-SPIONs in SCs.

Notes: Detection of cytotoxicity of SPIONs through LIVE/DEAD cell staining and the CCK-8 assay. Staining images of the LIVE/DEAD assay at 24 hrs (A-E) and 72 hrs (F-J) after transfection of SCs with different concentrations of SPIONs. (K) The percentage of dead cells was measured via the LIVE/DEAD assay. Live cells were stained green, while dead cells were stained red. (L) Cell viability was estimated using the CCK-8 assay. Scale bar: (A-J) $100 \mu \mathrm{m}$. Graph bars: mean \pm SD; $* * * p<0.005$ for comparison with $0 \mu$ g/mL group.

Abbreviations: CCK-8, cell counting Kit 8; PEI-SPIONs, polyethylenimine-coated superparamagnetic iron oxide nanoparticles; SCs, Schwann cells; SD, standard deviation.

\section{ChABC-Magnetofected SCs Secrete}

\section{Active Chondroitinase in vitro}

After incubation with conditioned medium from PEISPIONs/ChABC/SC (Figure 6B) cultures and purified ChABC (Figure 6C), the immunoreactivity for CS56 (to detect intact CSPGs) (green) in spinal cord sections was decreased compared with that observed in cells incubated with PBS (HyClone), especially in a dense area of astrocytes (Figure 6A). These results indicated that ChABC synthesized in PEI-SPIONs/ChABC/SCs were active. In addition, Immunofluorescence staining for CS56 and GFAP in astrocytes on the slide was performed to determine whether astrocytes could express CSPGs in vitro (Figure 6D-F). Notably, the DMB assay showed that enzymatic activity in conditioned medium from the PEI-
SPIONs/ChABC/SC culture was significantly higher (2.98 $\pm 0.46 \mathrm{mU} / \mu \mathrm{L} / 2 \mathrm{hr}$ ) than that measured in control cell culture medium $(0.81 \pm 0.23 \mathrm{mU} / \mu \mathrm{L} / 2 \mathrm{hr}, \mathrm{p}<0.001)$ or nontransduced SC cultures $(0.82 \pm 0.45 \mathrm{mU} / \mu \mathrm{L} / 2 \mathrm{hr}$, $\mathrm{p}<0.001$ ) (Figure 6G).

\section{Directed and Enhanced Migration of PEI-SPIONs/ChABC/SCs on an Astrocyte Monolayer Under an Applied MF}

The inverted coverslip migration assay was performed to investigate whether exogenous ChABC and PEI-SPIONs are capable of enhancing the migration of SCs over an astrocyte monolayer and manipulating the migration direction of SCs with an external MF. It was found that administration of MF or vector DNA magnetofection did not affect the migration of 


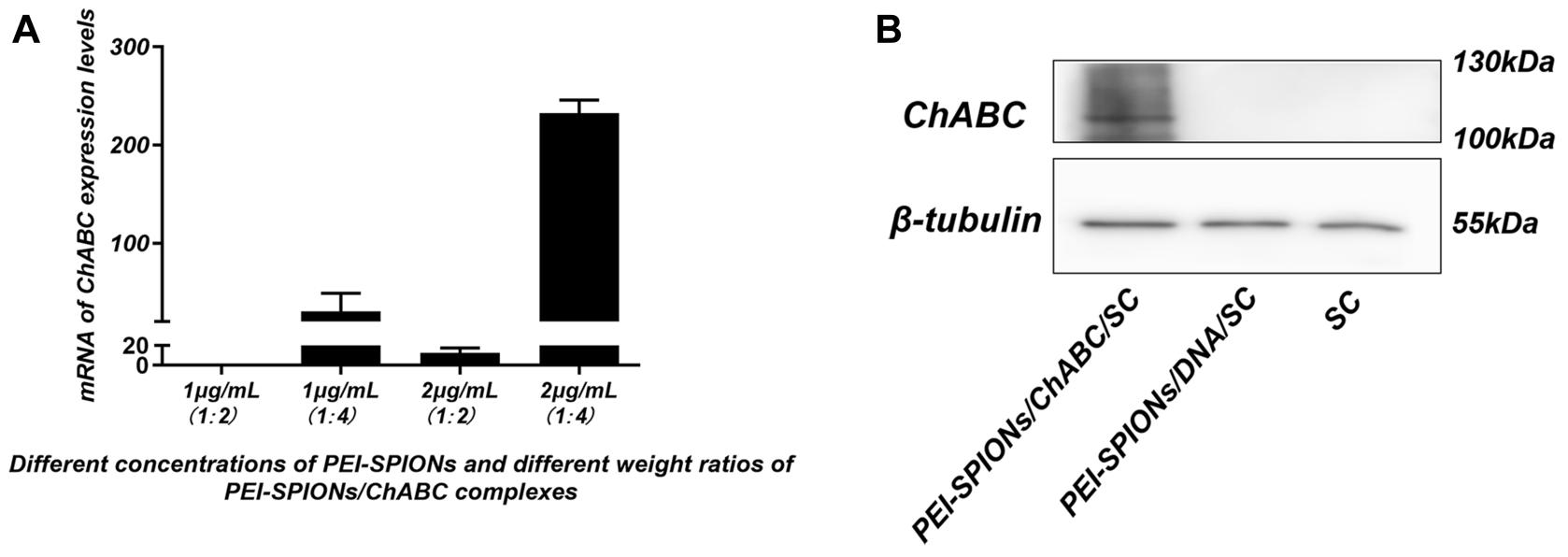

Figure 5 Expression of transferred exogenous ChABC in SCs.

Notes: (A) At 72 hrs after magnetofection, mRNA levels of ChABC in each group (normalized to normal control SCs). (B) At 72 hrs after magnetofection, detection of ChABC expression in SC, PEI-SPIONs/DNA/SC, and PEI-SPIONs/ChABC/SC by Western blotting.

Abbreviations: mRNA, messenger RNA; PEI-SPIONs, polyethylenimine-coated superparamagnetic iron oxide nanoparticles; ChABC, chondroitinase ABC; SCs, Schwann cells.

SCs on the astrocyte monolayer (Figure 7A-C). In contrast, the maximum migration distance of SCs was increased after magnetofection with $\mathrm{ChABC}$, but not in the desired direction (Figure 7E). When SCs magnetofected with PEI-SPIONs /DNA were exposed to an MF treatment, the maximal migration distance and orientation index were significantly enhanced (Figure 7D and G-I). More interestingly, when SCs with PEI-SPIONs/ChABC (PEI-SPIONs, $2 \mu \mathrm{g} / \mathrm{mL}$; PEISPION/ChABC, 1:4) were exposed to an MF treatment, their migration ability and orientation were further enhanced (Figure 7F and G-I), the magnetofected SCs preferentially migrated along the direction of the MF and migrated into the
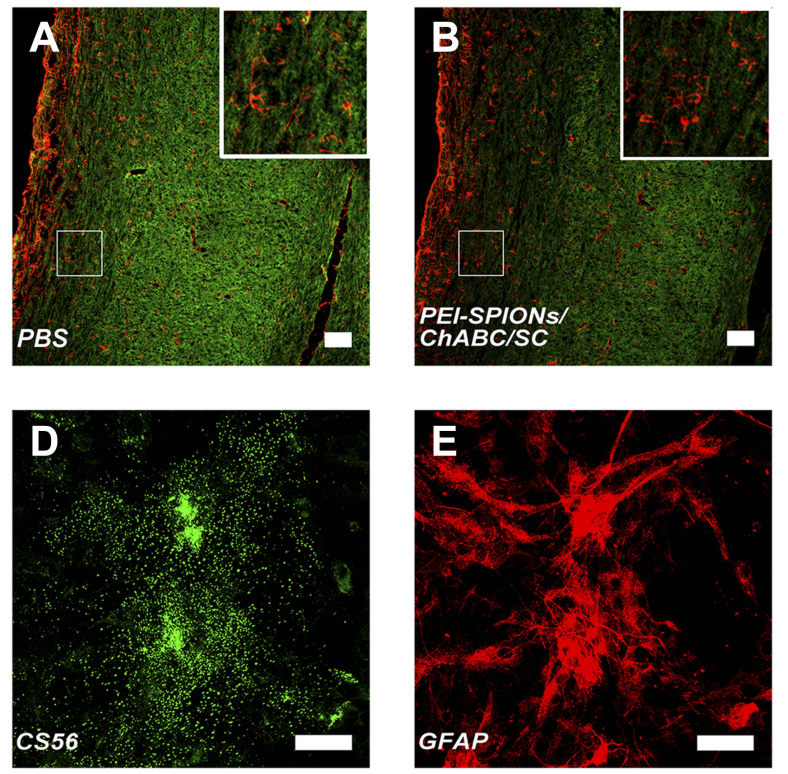
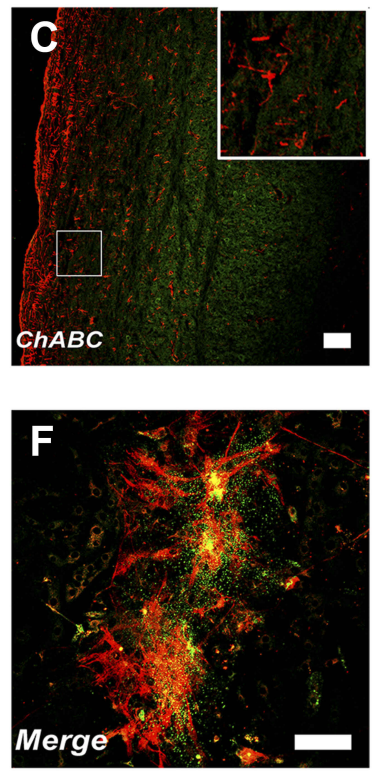

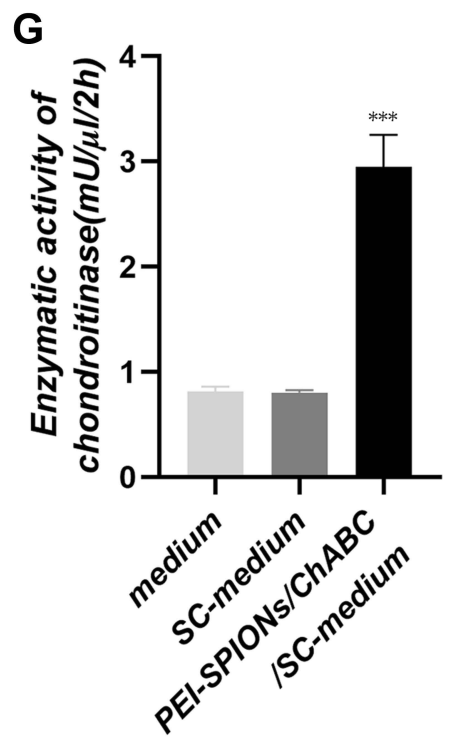

Figure 6 Activity and function assay of ChABC expressed by magnetofected SCs in vitro.

Notes: (A-C) After CS56 (green) and GFAP (red) staining, the immunoreactivity in spinal cord sections incubated with conditioned medium from ChABC-magnetofected SC culture (B) and purified ChABC (C) was decreased compared with that observed in cells treated with PBS (A). (D-F) After CS56 (green), GFAP (red) staining and merge file, the immunoreactivity in a slide of astrocytes. (G) The DMB assay demonstrated the enzymatic activity of ChABC in conditioned medium from PEI-SPIONs/ChABC/SC cultures, control cell culture medium or nontransduced SC cultures. Data were presented as the mean \pm SEM; $n=6$ per each SC group. ${ }^{* * *} p<0.005$ for comparison with medium group. Scale bar (A-C), 100 $\mu \mathrm{m}$. Scale bar (D-F), $50 \mu \mathrm{m}$.

Abbreviations: GFAP, glial fibrillary acidic protein; CS56, monoclonal anti-chondroitin sulfate; PEI-SPIONs, polyethylenimine-coated superparamagnetic iron oxide nanoparticles; ChABC, chondroitinase ABC; SCs, Schwann cells. 
astrocyte monolayer. The orientation index in the PEISPIONs/ChABC/SCs $+\mathrm{MF}$ group was significantly higher than that determined in the remaining groups (Figure 7G). In addition, the maximum migration distance was greatest in the PEI-SPIONs/ChABC/SCs + MF group $(1,469.47 \pm 196.00 \mu \mathrm{m})$, followed by the PEI-SPIONs/DNA/SCs+MF group (736.31 $\pm 252.17 \mu \mathrm{m})$, the PEI-SPIONs/ChABC/SCs group (318.15 $\pm 197.28 \mu \mathrm{m})$, and the remaining groups. Approximately $80-85 \%$ of the cells move in the $800 \mu \mathrm{m}$ range. Therefore, we counted the number of cells which migrated within 800 $\mu \mathrm{m}$. It was found that the number of SCs in the PEI-SPIONs $/ \mathrm{ChABC} / \mathrm{SCs}+\mathrm{MF}$ group $\quad(61.50 \pm 7.30 \quad$ cells $/ 800 \quad \mu \mathrm{m}$; Figure 7J) was significantly higher than that recorded in the PEI-SPIONs/ChABC/SCs without MF $(30.83 \pm 2.79$ cells/800 $\mu \mathrm{m}$; Figure 7J). Moreover, the number of SCs at a distance of $200 \mu \mathrm{m}$ from the edge in the PEI-SPIONs/ChABC/SCs $+\mathrm{MF}$, PEI-SPIONs/DNA/SCs+MF and PEI-SPIONs/ChABC/SCs groups was 1.52-, 1.32-, and 1.17- fold than that reported in the control SC group, respectively. Similarly, the number of $\mathrm{SCs}$ in the PEI-SPIONs/ChABC/SCs+MF, PEI-SPIONs /DNA/SCs $+\mathrm{MF}$ and PEI-SPIONs/ChABC/SCs groups was 2.12-, 1.77-, and 1.23-fold higher at distances of $400 \mu \mathrm{m}$, and 2.70-, 2.21-, and 1.30-fold higher at distances of 600 $\mu \mathrm{m}$ than that observed in the control SC group (Figure 7J). In the PEI-SPIONs/ChABC/SCs + MF groups, the maximal distance of SC migration was $1,800 \mu \mathrm{m}$ (Figure 7J), confirming that PEI-SPIONs/ChABC/SCs fused well with astrocytes. Collectively, these results indicate that ChABC magnetofection is capable of enhancing the migration of SCs over an astrocyte monolayer and manipulating the direction of $\mathrm{SC}$ migration with an external MF.

\section{PEI-SPIONs/ChABC/SCs Crossed Boundaries When in Contact with Astrocytes and Their Direction Was Oriented by an Applied MF}

The confrontation assay was performed to investigate whether PEI-SPIONs/ChABC/SCs could migrate through the astrocyte boundary and migrate into the astrocyte domain (Figure 8). A schematic diagram of the confrontation assay is shown in Figure 8G. Cell boundaries were generated between astrocytes and SCs in control SCs, nonmagnetofected SCs + MF and PEISPIONs/DNA/SCs (Figure 8A-C). Cells in the PEI-SPIONs /DNA/SCs + MF and PEI-SPIONs/ChABC/SCs without or with an external MF were capable of crossing the boundary formed by astrocytes (Figure 8D-F). Under the guidance of MF, SCs migrated along the direction of the MF axis in PEI-SPIONs
/DNA/SCs and PEI-SPIONs/ChABC/SCs (Figure 8D and F). In addition, the number of SCs which crossed the boundary and migrated into the region of astrocytes was highest in the PEISPIONs/ChABC/SCs+MF group, followed by the PEISPIONs/DNA/SCs+MF group, the PEI-SPIONs/ChABC/SC group, and the remaining three groups (Figure 8H).

\section{Discussion}

In our previous study, we have demonstrated that PEISPIONs/DNA/SCs could be driven by an applied MF to cross the cell boundary and migrate into the astrocyte domain. However, the maximum distance of PEI-SPIONs /DNA/SC migration is limited by their poor migration when in contact with astrocytes, ${ }^{14}$ It has been reported that a glial scar formed by astrocytes is capable of preventing SCs from passing through the boundary and migrating into the astrocyte area, which significantly limits the application of SCs in the repair of SCI. ${ }^{39,40}$ In the present study, genetically modified $\mathrm{Ch} A B C$ was introduced into SCs through magnetofection to stably express ChABC, which is capable of reducing the production of CSPGs by activated astrocytes. ${ }^{19}$ It was found that the number of cells migrating into the astrocyte region and the maximum migration distance was significantly increased in PEI-SPIONs/ChABC/ $\mathrm{SCs}$, especially when the cells were exposed to a stable MF. The results of this study proposed a new approach to guide and enhance the migration of PEI-SPIONs/ChABC/SCs within an astrocyte micro-environment with the aid of an applied MF. These findings highlight the application of these cells in the field of neuranagenesis in CNS.

Magnetofection offers the advantages of simplicity, high efficiency and low toxicity over other transfection methods. ${ }^{26,27}$ It has been shown that the expression efficiency of lipofectamine sharply decreased to $40.32 \%$ at $48 \mathrm{hrs}$. In contrast, the efficiency of magnetofection reached $76.6 \%$ at $72 \mathrm{hrs}$ and maintained a high level $(72.3 \%)$ at $120 \mathrm{hrs}^{27}$ Therefore, we selected magnetofection to mediate the entry of the plasmid into SCs. As a gene vector in magnetofection, SPIONs have been widely used owing to their safety profile, low toxicity, high stability, and high efficiency of transfection. Currently, SPIONs can be artificially driven by an external MF; this approach has been used in drug release, cell separation, and magnetic resonance imaging. ${ }^{41,42}$ However, studies showed that in the absence of an appropriate coating nanoparticles tend to form irreversible agglomerates. ${ }^{43}$ Therefore, it is essential to modify SPIONs with polymers to increase their stability and manipulate their surface properties to improve cell and material interface. ${ }^{44,45}$ Base on the reason above, it is important 

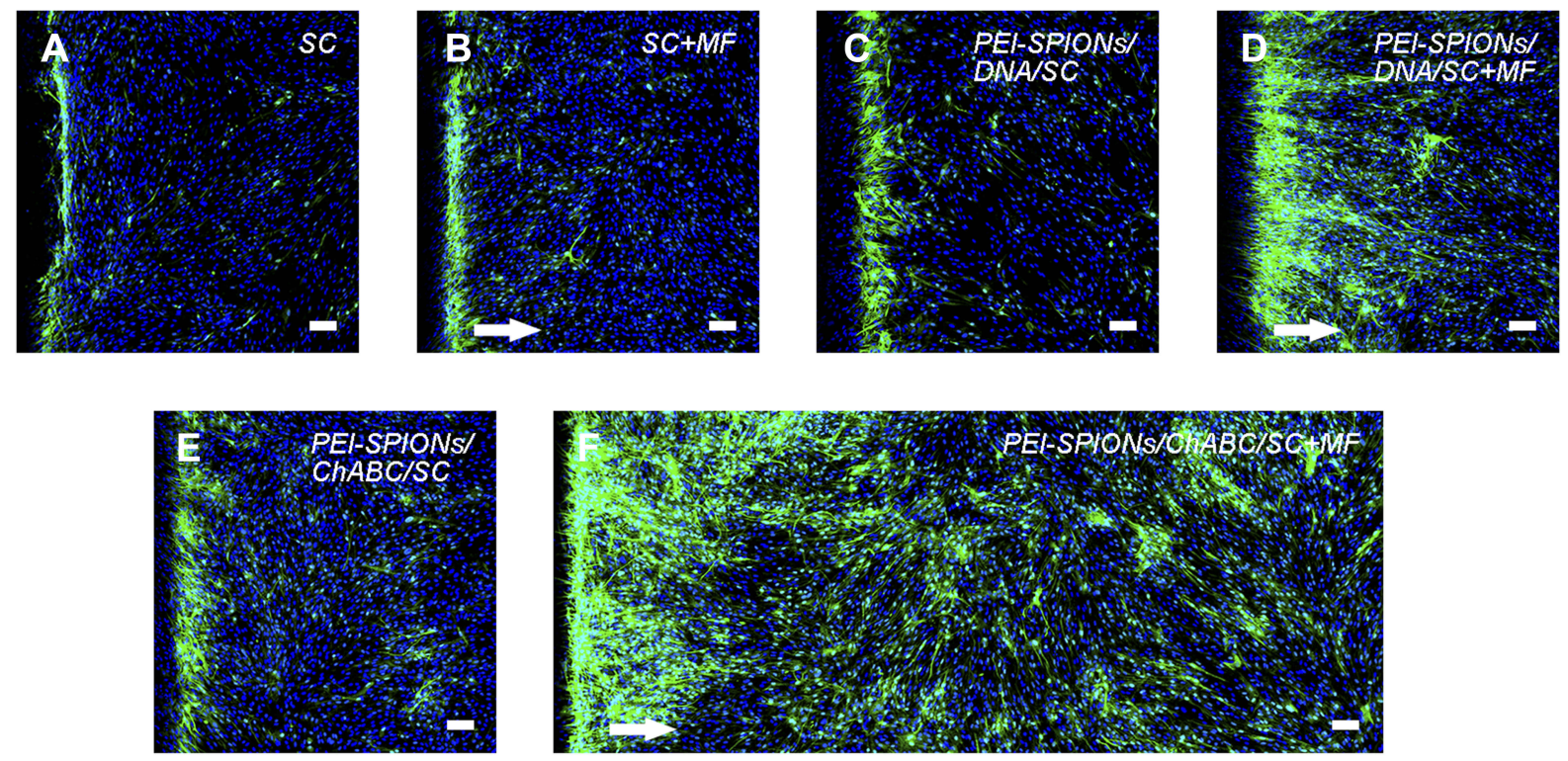

G

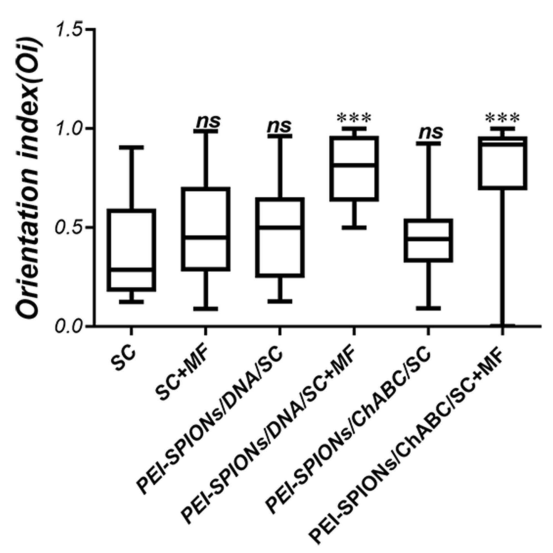

I

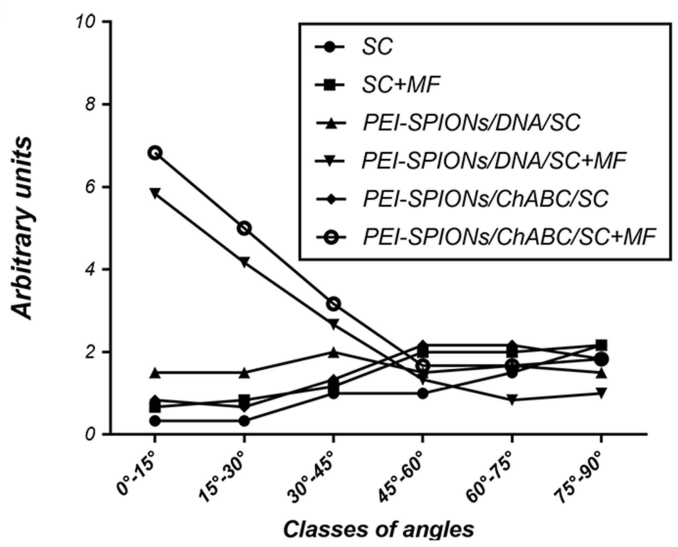

H

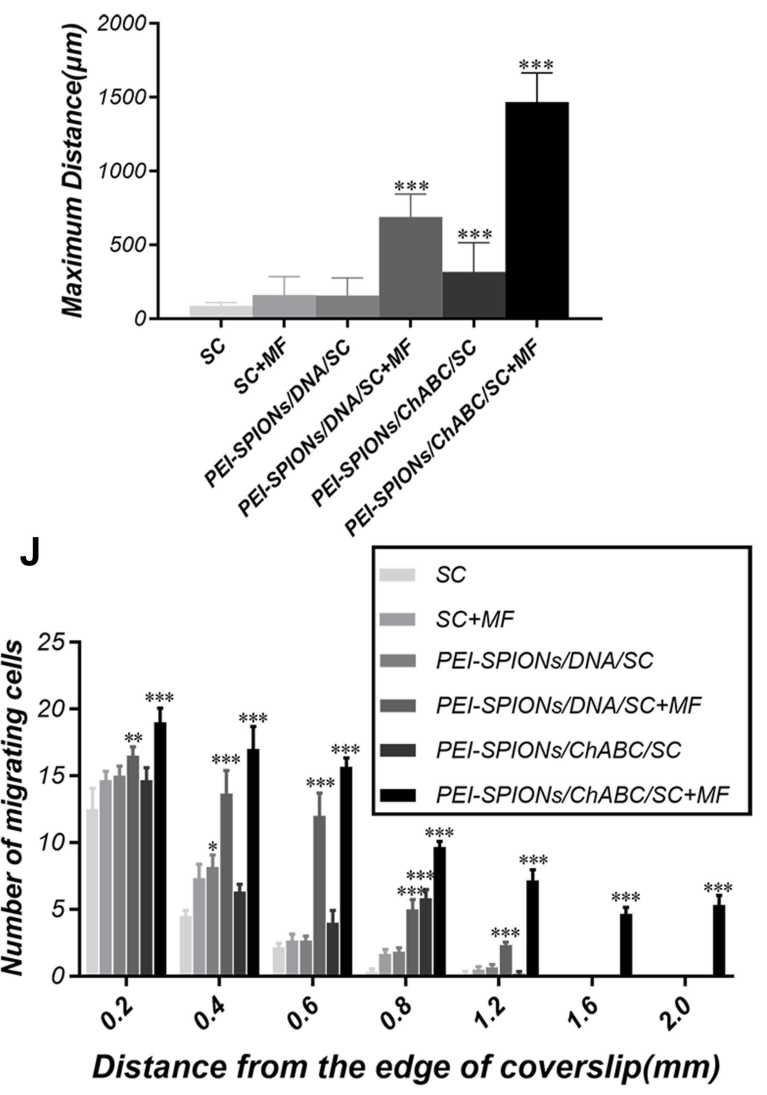

Figure 7 Typical images of SC migration on an astrocyte monolayer in the inverted coverslip migration assay.

Notes: (A-F) SCs were labeled with SI00 (green) and DAPI (blue), while astrocytes were labeled with the nucleus staining solution DAPI (blue) only. White arrows indicate the direction of the applied MF (F) The migration of PEI-SPIONs/ChABC/SC on the astrocyte monolayer was significantly enhanced and oriented parallel to the direction of the magnetic force in the presence of an external MF. (G) SC migration orientation index. The box-plot showed the median, interquartile ranges, maximum and minimum. (H) Average maximum distances of migration from the edge of the inverted fragments. (I) Number of SCs (normalized to the control SC) grouped into classes of angles formed by the long axis of SCs and the direction of the external MF. (J) Number of SCs reaching different specific distances from the edge of an inverted fragment. Scale bar: (A-F) I00 $\mu \mathrm{m}$. Graph bars: mean \pm SD; ${ }^{*}<<0.05$, $* * p<0.01$, ***p $<0.005$ for comparison with SC group.

Abbreviations: DAPI, 4',6-diamidino-2-phenylindole; MF, magnetic field; ns, not significant; PEI-SPIONs, polyethylenimine-coated superparamagnetic iron oxide nanoparticles; ChABC, chondroitinase ABC; SCs, Schwann cells; SD, standard deviation. 

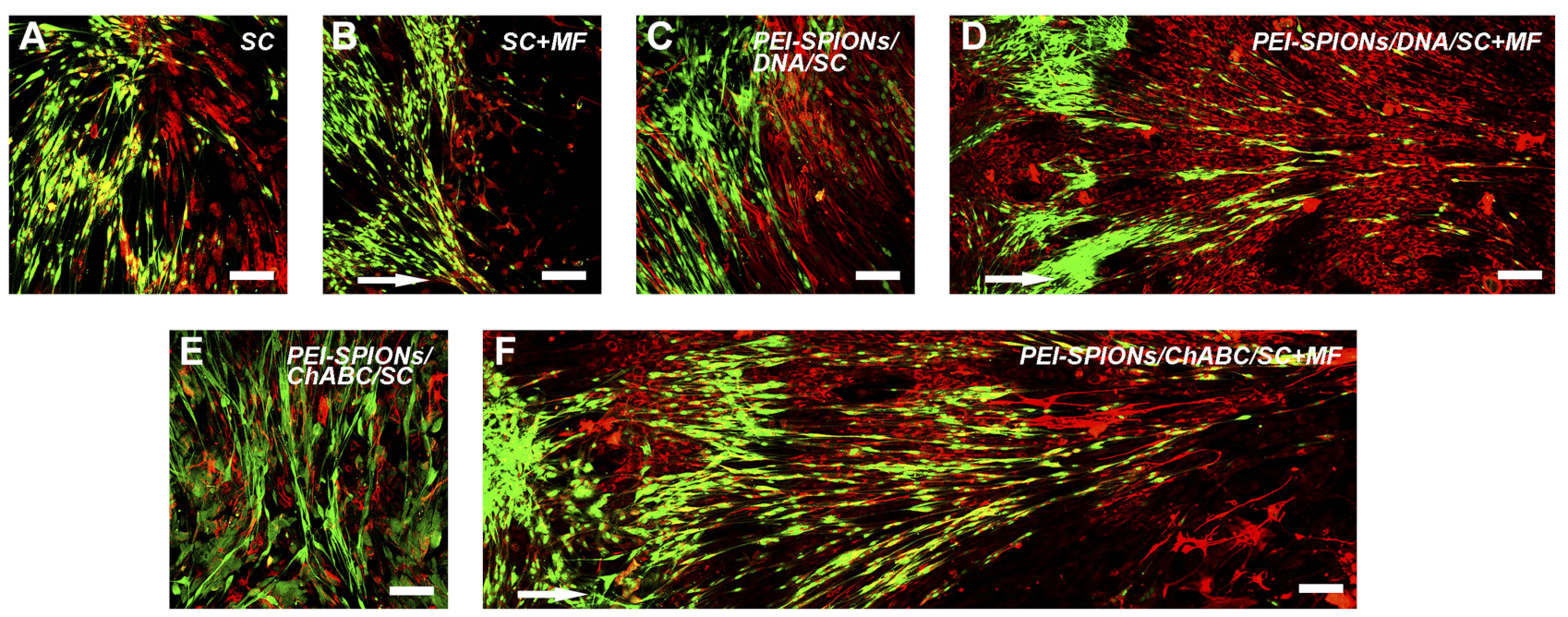

G

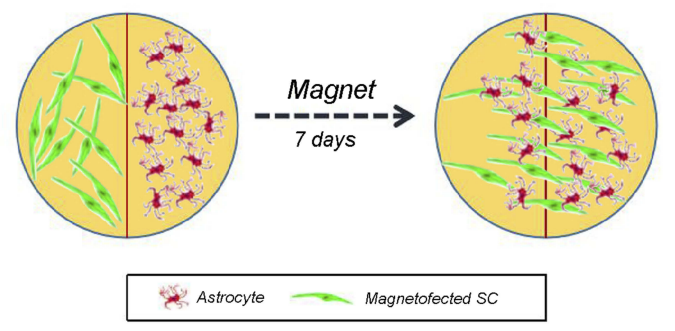

H

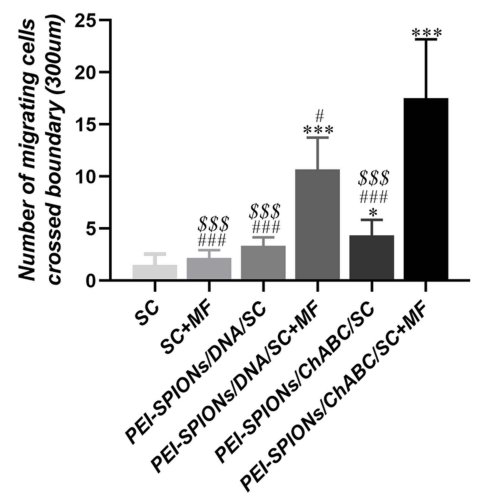

Figure 8 Confrontation assays of SCs and astrocytes.

Notes: Immunocytochemistry images of the SC-astrocyte confrontation assay. SCs were labeled with SI00 (green); while astrocytes were labeled with GFAP (red). (A-F) Arrows indicated the direction of the MF. (A, B) SC with or without an applied MF; boundaries were formed by astrocytes; (C) PEI-SPIONs/DNA/SC formed a boundary with astrocytes in the absence of an applied MF; (D) PEI-SPIONs/DNA/SC crossed the cell boundary in the presence of an MF. (E) PEI-SPIONs/ChABC/SC migrated into the astrocyte domain without a specific direction. (F) PEI-SPIONs/ChABC/SC broke the boundary formed with astrocytes and aligned preferentially along the axis of the magnetic force under an applied MF. (G) Schematic diagram of the SC-astrocyte confrontation assay. Magnetofected SCs and astrocytes were plated in parallel strips; both were allowed to grow and migrate for 7 days under an applied MF. $(\mathbf{H})$ Quantification of SCs that crossed the boundary and migrated into the astrocyte domain. The number of PEI-SPIONs/ChABC/SC with an applied MF far exceeded those of the other groups. Scale bar: (A-F) $100 \mu \mathrm{m}$. Graph bars: mean \pm SD; $* p<0.05$, *** $p<0.005$ for comparison with SC group; ${ }^{\#}<0.05,{ }^{\#<}<0.005$ for comparison with PEI-SPIONS/ChABC/SC+MF group; ${ }^{\$ \$} \$<0.005$ for comparison with PEI-SPIONS/DNA/SC+MF group.

Abbreviations: GFAP, glial fibrillary acidic protein; MF, magnetic field; ns, not significant; PEI-SPIONs, polyethylenimine-coated superparamagnetic iron oxide nanoparticles; ChABC, chondroitinase ABC; SCs, Schwann cells; SD, standard deviation.

to functionalize SPIONs to achieve a positive charged surface to facilitate cell endocytosis and avoid aggregating in our study. Polyethylenimine (PEI) is a widely used cationic polymer to modify the surface of SPIONs. Furthermore, the internalized PEI-coated SPIONs (PEI-SPIONs) can exert cellular tension under an applied MF, which sufficiently initiates axonal outgrowth and cell migration in the desired direction. The PEISPIONs in the present study were synthetized by the company of Chemicell. Compared with SPIONs, the tendency to aggregate of PEI-SPIONs has been significantly improved. In addition, the diameter of PEI-SPIONs allows their endocytosis by the cells. In the present study, the ChABC exogenous gene was successfully expressed in cells after magnetofection with ChABC-loaded PEI-SPIONs. The surface of SPIONs is positively charged by the attachment of a polymer (eg, PEI), which is capable of adhering to cells to further increase the distribution of SPIONs on the cell membrane and internalization. ${ }^{46,47}$ In our study, the PEI-SPIONs/DNA complex adhered to the cells and was rapidly internalized under the action of an external MF after magnetofection. The presence of PEI-SPIONs has been reported on the cell surface and within the cells. The difference between the average diameter of PEI-SPIONs determined through TEM and ZetaPALS may be attributed to the agglomeration of charged PEI-SPIONs in the preparation of the sample 
solution for ZetaPALS. ${ }^{48,49}$ We also found that the toxicity of PEI-SPIONs is dose and time dependent. The SPIONs were less toxic to the primary SCs at concentrations $<4 \mu \mathrm{g} / \mathrm{mL}$. Therefore, in the present study, we selected $2 \mu \mathrm{g} / \mathrm{mL}$ as a safe experimental concentration.

The use of a static MF to guide the direction of cell migration has been widely investigated, especially within the vascular system. ${ }^{50,51}$ Previous studies have reported that cell migration can be enhanced by internalized magnetic nanoparticles in the presence of an $\mathrm{MF}^{3,31}$ In the present study, we applied an external MF to SCs after internalization of SPIONs. We firstly showed that the migration ability and migration direction of SCs were not affected by the magnetofection of SPIONs in the absence of an external MF. The migration of SCs with magnetofected SPIONs was significantly enhanced after the application of a static MF. More interestingly, the SCs preferentially migrated along the axis of the MF, indicating that the MF is capable of enhancing the migration of SCs and guiding its direction with the aid of internalized SPIONs. This ability may be of great value for $\mathrm{SCs}$ to improve their performance in nerve injury repair.

Although the migration of SCs with internalize magnetic SPIONs was enhanced by the external MF, the glial scar formed by astrocytes remains still a strong hindrance to the migration of SCs. ${ }^{19}$ Previous studies have shown that CSPGs interact with the transmembrane receptor protein tyrosine phosphatase in axons and inhibit their growth in the injured spinal cord; this effect can be inhibited by treatment with ChABC. Numerous studies have demonstrated that both transfection of a genetically modified $\mathrm{ChABC}$ gene into cells or exogenous $\mathrm{ChABC}$ showed marked effects on the degradation of CSPGs in glial scars ${ }^{33}$ and enhanced the regeneration of transected propriospinal axons after spinal cord injury. However, local injection of $\mathrm{ChABC}$ is limited by the requirement for long-term infusion or repeated injections, which are associated with risks of tissue injury, immunogenicity, and infection. $^{20,22}$ In this case, delivery of a vector containing the $\mathrm{ChABC}$ gene into glial cells may be an alternative solution. However, $\mathrm{ChABC}$ is a bacterial enzyme obtained from Proteus vulgaris, and its expression in mammalian cells is problematic. When the newly synthesized chondroitin peptide is secreted through eukaryotic cells, the hidden $\mathrm{N}$-glycosylation sites become glycosylated, potentially interfering with the folding and secretion of ChABC. It has been shown that this process leads to failure of functional enzyme production in mammalian cells from the original bacterial chondroitinase gene. Therefore, the chondroitin enzyme gene has been modified through directional mutation of up to six selected $\mathrm{N}$-glycosylation sites, which are located in important positions of structural or ligand binding. These mutations did not affect the enzyme activity in vitro. In the present study, the modified chondroitin enzyme gene was transfected into SCs, and our data showed that PEI-SPIONs/ChABC/SCs (PEI-SPIONs, $2 \mu \mathrm{g}$ / $\mathrm{mL}$; PEI-SPION/ChABC, 1:4) up-regulated the mRNA levels of ChABC by 232.4-fold versus those measured in control SCs. Furthermore, Western blotting analysis of PEI-SPIONs /ChABC/SC showed that the $\mathrm{ChABC}$ protein was successfully expressed. We treated the normal spinal cord with the supernatant from the PEI-SPIONs/ChABC/SCs to demonstrate the activity of the enzyme. Many studies reported that CSPGs are a class of extracellular matrix proteoglycans widely expressed within the CNS; they can be synthesized by all types of neural cells. $^{52,53}$ In this study, we also found that CSPGs were expressed in the normal spinal cord tissue. In previous studies, CSPGs were highly expressed in the site of spinal cord injury. ${ }^{54,55}$ In the present study, we found that the fluorescence intensity of CS56 has been decreased after the supplementation of pure $\mathrm{ChABC}$ into the spinal cord section. In addition, the fluorescence intensity of CS56 of the spinal cord section was also decreased by the culture medium of PEI-SPIONs/ChABC/ SCs. Collectively, the results demonstrated that $\mathrm{ChABC}$ protein expressed by PEI-SPIONs/ChABC/SCs is biologically active, which is capable of degrading CSPGs of spinal cord tissues. As an extracellular matrix component of the CNS, CSPGs are secreted by astrocytes. The representative confocal images of astrocyte cultures with GFAP (red) and CS56 (green) clearly demonstrated that CSPGs were located within and outside the astrocytes. The reduction of CSPGs in the area of the injured spinal cord is beneficial for promoting Schwann cell migration and nerve regeneration. Subsequently, the orientation of SC migration was effectively guided by the directional force of an external MF on internalized SPIONs. Therefore, the synergistic effects of the directional force and enhanced degradation of CSPGs by ChABC contribute to the migration of SCs into the astrocyte area.

In the present study, we firstly magnetofeced SCs using ChABC-loaded PEI-SPIONs to up-regulate the expression of ChABC, which was capable of decreasing the CSPGs secreted by astrocytes. With the directional force of an external MF on internalized SPIONs within SCs, both the number of migrated cells and the migration distance of PEI-SPIONS/ChABC/SCs were significantly increased within the astrocyte area. As the main component of a glial scar formed locally in spinal cord injury, CSPGs inhibit axonal regeneration. ${ }^{21}$ In this study, we demonstrated that ChABC from PEI-SPIONs/ChABC/SCs is bioactive and capable of degrading CSPGs in the spinal cord tissue. Moreover, the reduction of CSPGs in the area of the 
injured spinal cord is helpful in promoting SC migration and nerve regeneration. ${ }^{18}$ Additional studies will investigate whether PEI-SPIONs/ChABC/SCs are capable of secreting bioactive $\mathrm{ChABC}$ and degrading the CSPGs in an injured spinal cord animal model. Furthermore, the effect of PEISPIONs/ChABC/SCs on promoting nerve repair will also be evaluated in vivo in our future study. Notably, the mechanism through which the MF acts on the force of the SCs using SPIONs remains to be elucidated. More importantly, further studies are warranted to optimize the protocol for grafting magnetofected SCs into injury lesions in animal models of SCI, as well as the application of MFs to evaluate their effect on glial scar formation and axonal regeneration.

\section{Conclusion}

The results of the present study demonstrated that SPIONsmediated magnetofection of $\mathrm{ChABC}$ is capable of upregulating the expression of $\mathrm{ChABC}$ and enhancing the migration of SCs in an astrocyte-rich environment. Using an MF, the migration direction of PEI-SPIONS/ChABC/SCs was guided along the magnetic axis, leading to the fusion of magnetic SCs into astrocytes. These results raise the possibility that magnetically functionalized SCs migrate in a specific direction in astrocyte-rich injury lesions and establish an SC bridge to guide the direction of axonal regeneration to their distal destination in the CNS. This hypothesis warrants further investigation in future in vivo studies.

\section{Acknowledgments}

This work was supported by the National Basic Research Program of China (973 Program no 2014CB542206), the National Natural Science Foundation of China (Grant nos. 81672148, 81730065, 81601903 and 81802143), and Excellent Doctoral Thesis of China (Grant no. 201480). We thank Chemicell (Berlin, Germany) for kindly providing information on the magnetization of PEI-SPIONs. We also thank Lifeng Lan, Jing Liu, and Xiwang Hu, for their excellent technical assistance.

\section{Disclosure}

The authors report no conflicts of interest in this work.

\section{References}

1. Wang X, Peng C, Zhang S, et al. Polysialic-acid-based micelles promote neural regeneration in spinal cord injury therapy. Nano Lett. 2019;19(2):829-838. doi:10.1021/acs.nanolett.8b04020

2. Tran AP, Warren PM, Silver J. The biology of regeneration failure and success after spinal cord injury. Physiol Rev. 2018;98(2):881-917. doi:10.1152/physrev.00017.2017
3. Huang L, Xia B, Liu Z, Cao Q, Huang J, Luo Z. Superparamagnetic iron oxide nanoparticle-mediated forces enhance the migration of schwann cells across the astrocyte-schwann cell boundary in vitro. Front Cell Neurosci. 2017;11:83.

4. Arthur-Farraj PJ, Latouche M, Wilton DK, et al. c-Jun reprograms Schwann cells of injured nerves to generate a repair cell essential for regeneration. Neuron. 2012;75(4):633-647. doi:10.1016/j.neuron.2012.06.021

5. Huang J, Hu X, Lu L, Ye Z, Zhang Q, Luo Z. Electrical regulation of Schwann cells using conductive polypyrrole/chitosan polymers. J Biomed Mater Res A. 2009;9999A(1):NA-NA. doi:10.1002/jbm. a.32511

6. Papastefanaki F, Chen J, Lavdas AA, Thomaidou D, Schachner M, Matsas R. Grafts of Schwann cells engineered to express PSA-NCAM promote functional recovery after spinal cord injury. Brain. 2007;130(8):2159-2174. doi:10.1093/brain/awm155

7. Houle JD, Tom VJ, Mayes D, Wagoner G, Phillips N, Silver J. Combining an autologous peripheral nervous system "bridge" and matrix modification by chondroitinase allows robust, functional regeneration beyond a hemisection lesion of the adult rat spinal cord. $J$ Neurosci. 2006;26(28):7405-7415. doi:10.1523/JNEUROSCI.116606.2006

8. Dezawa M, Adachi-Usami E. Role of Schwann cells in retinal ganglion cell axon regeneration. Prog Retin Eye Res. 2000;19(2):171-204. doi:10.1016/S1350-9462(99)00010-5

9. Liu Z, Zhu S, Liu L, et al. A magnetically responsive nanocomposite scaffold combined with Schwann cells promotes sciatic nerve regeneration upon exposure to magnetic field. Int $J$ Nanomedicine. 2017;12:7815-7832. doi:10.2147/IJN

10. Bunge MB. Novel combination strategies to repair the injured mammalian spinal cord. J Spinal Cord Med. 2016;31(3):262-269. doi:10.1080/10790268.2008.11760720

11. Kanno H, Pearse DD, Ozawa H, Itoi E, Bunge MB. Schwann cell transplantation for spinal cord injury repair: its significant therapeutic potential and prospectus. Rev Neurosci. 2015;26(2):121-128. doi:10.1515/revneuro-2014-0068

12. Tetzlaff W, Okon EB, Karimi-Abdolrezaee S, et al. A systematic review of cellular transplantation therapies for spinal cord injury. J Neurotrauma. 2011;28(8):1611-1682. doi:10.1089/neu.2009.1177

13. Fortun J, Hill CE, Bunge MB. Combinatorial strategies with Schwann cell transplantation to improve repair of the injured spinal cord. Neurosci Lett. 2009;456(3):124-132. doi:10.1016/j.neulet.2008.08.092

14. Chaudhry N, Bachelin C, Zujovic V, et al. Myelin-associated glycoprotein inhibits schwann cell migration and induces their death. J Neurosci. 2017;37(24):5885-5899. doi:10.1523/JNEUROSCI.1822-16.2017

15. Grimpe B, Pressman Y, Bunge MB, Silver J. The role of proteoglycans in Schwann cell/astrocyte interactions and in regeneration failure at PNS/CNS interfaces. Mol Cell Neurosci. 2005;28(1):18-29. doi:10.1016/j.men.2004.06.010

16. Lakatos A, Barnett SC, Franklin RJM. Olfactory ensheathing cells induce less host astrocyte response and chondroitin sulphate proteoglycan expression than schwann cells following transplantation into adult CNS white matter. Exp Neurol. 2003;184(1):237-246. doi:10.1016/S0014-4886(03)00270-X

17. Wiliams RR, Bunge MB. Schwann cell transplantation: a repair strategy for spinal cord injury? Prog Brain Res. 2012;201:295-312.

18. Koh CH, Pronin S, Hughes M. Chondroitinase ABC for neurological recovery after acute brain injury: systematic review and meta-analyses of preclinical studies. Brain Injury. 2018;32 (6):715-729. doi:10.1080/02699052.2018.1438665

19. Takeuchi K, Yoshioka N, Higa Onaga S, et al. Chondroitin sulphate $\mathrm{N}$-acetylgalactosaminyl-transferase-1 inhibits recovery from neural injury. Nat Commun. 2013;4(1). doi:10.1038/ncomms3740.

20. Zhao R, Muir EM, Alves JN, et al. Lentiviral vectors express chondroitinase $\mathrm{ABC}$ in cortical projections and promote sprouting of injured corticospinal axons. J Neurosci Meth. 2011;201(1):228-238. doi:10.1016/j.jneumeth.2011.08.003 
21. Chen M, Zheng B. Axon plasticity in the mammalian central nervous system after injury. Trends Neurosci. 2014;37(10):583-593. doi:10.1016/j.tins.2014.08.008

22. Muir EM, Fyfe I, Gardiner S, et al. Modification of N-glycosylation sites allows secretion of bacterial chondroitinase ABC from mammalian cells. $J$ Biotechnol. 2010;145(2):103-110. doi:10.1016/j. jbiotec.2009.11.002

23. Buss A. Gradual loss of myelin and formation of an astrocytic scar during Wallerian degeneration in the human spinal cord. Brain. 2004;127(1):34-44. doi:10.1093/brain/awh001

24. Iwashita Y, Fawcett JW, Crang AJ, Franklin RJM, Blakemore WF. Schwann cells transplanted into normal and X-irradiated adult white matter do not migrate extensively and show poor long-term survival. Exp Neurol. 2000;164(2):292-302. doi:10.1006/exnr.2000.7440

25. Amani H, Mostafavi E, Arzaghi H, et al. Three-dimensional graphene foams: synthesis, properties, biocompatibility, biodegradability, and applications in tissue engineering. Acs Biomater Sci Eng. 2019;5 (1):193-214. doi:10.1021/acsbiomaterials.8b00658

26. Zhang X, Wu F, Men K, et al. Modified Fe3O4 magnetic nanoparticle delivery of $\mathrm{CpG}$ inhibits tumor growth and spontaneous pulmonary metastases to enhance immunotherapy. Nanoscale Res Lett. 2018;13 (1). doi:10.1186/s11671-018-2661-8.

27. Zhao X, Cui H, Chen W, et al. Morphology, structure and function characterization of PEI modified magnetic nanoparticles gene delivery system. PLoS One. 2014;9(6):e98919. doi:10.1371/journal. pone.0098919

28. Krötz F, Wit CD, Sohn H, et al. Magnetofection-A highly efficient tool for antisense oligonucleotide delivery in vitro and in vivo. $\mathrm{Mol}$ Ther. 2003;7(5):700-710. doi:10.1016/S1525-0016(03)00065-0

29. Lopez-Verrilli MA, Picou F, Court FA. Schwann cell-derived exosomes enhance axonal regeneration in the peripheral nervous system. Glia. 2013;61(11):1795-1806. doi:10.1002/glia.22558

30. Afshari FT, Fawcett JW. Astrocyte-Schwann-cell coculture systems. Methods Mol Biol. 2012;814:381-391.

31. Xia B, Huang L, Zhu L, et al. Manipulation of Schwann cell migration across the astrocyte boundary by polysialyltransferase-loaded superparamagnetic nanoparticles under magnetic field. Int $J$ Nanomed. 2016;11:6727-6741. doi:10.2147/IJN.S122358

32. Liu T, Xu J, Chan BP, Chew SY. Sustained release of neurotrophin-3 and chondroitinase $\mathrm{ABC}$ from electrospun collagen nanofiber scaffold for spinal cord injury repair. J Biomed Mater Res A. 2012;100A (1):236-242. doi:10.1002/jbm.a.v100a.1

33. Kanno H, Pressman Y, Moody A, et al. Combination of engineered Schwann cell grafts to secrete neurotrophin and chondroitinase promotes axonal regeneration and locomotion after spinal cord injury. $J$ Neurosci. 2014;34(5):1838-1855. doi:10.1523/JNEUROSCI.266113.2014

34. Moon LDF, Asher RA, Rhodes KE, Fawcett JW. Relationship between sprouting axons, proteoglycans and glial cells following unilateral nigrostriatal axotomy in the adult rat. Neuroscience. 2002;109(1):101-117. doi:10.1016/S0306-4522(01)00457-2

35. Afshari FT, Kwok JC, Fawcett JW. Analysis of Schwann-astrocyte interactions using in vitro assays. $J$ Visualized Exp. 2011;(47):2214.

36. Cao L, Zhu YL, Su Z, et al. Olfactory ensheathing cells promote migration of Schwann cells by secreted nerve growth factor. Glia. 2007;55(9):897-904. doi:10.1002/glia.20511

37. O’Neill P, Lindsay SL, Pantiru A, et al. Sulfatase-mediated manipulation of the astrocyte-Schwann cell interface. Glia. 2017;65 (1):19-33. doi:10.1002/glia.23047

38. Ma T, Wang Y, Qi F, et al. The effect of synthetic oxygen carrier-enriched fibrin hydrogel on Schwann cells under hypoxia condition in vitro. Biomaterials. 2013;34(38):10016-10027. doi:10. 1016/j.biomaterials.2013.09.047

39. Okada S, Hara M, Kobayakawa K, Matsumoto Y, Nakashima Y. Astrocyte reactivity and astrogliosis after spinal cord injury. Neurosci Res. 2018;126:39-43. doi:10.1016/j.neures.2017.10.004
40. Silver J, Miller JH. Regeneration beyond the glial scar. Nat Rev Neurosci. 2004;5(2):146-156. doi:10.1038/nrn1326

41. Prabha G, Raj V. Preparation and characterization of chitosan-Polyethylene glycol-polyvinylpyrrolidone-coated superparamagnetic iron oxide nanoparticles as carrier system: drug loading and in vitro drug release study. J Biomed Mat Res Part B. 2016;104 (4):808-816. doi:10.1002/jbm.b.v104.4

42. Cicha I, Scheffler L, Ebenau A, Lyer S, Alexiou C, Goppelt-Struebe M. Mitoxantrone-loaded superparamagnetic iron oxide nanoparticles as drug carriers for cancer therapy: uptake and toxicity in primary human tubular epithelial cells. Nanotoxicology. 2015;10(5):557-566. doi:10.3109/17435390.2015.1095364

43. Bilyy R, Unterweger H, Weigel B, et al. Inert coats of magnetic nanoparticles prevent formation of occlusive intravascular co-aggregates with neutrophil extracellular traps. Front Immunol. 2018;9. doi:10.3389/fimmu.2018.02266.

44. Amani H, Kazerooni H, Hassanpoor H, Akbarzadeh A, PazokiToroudi H. Tailoring synthetic polymeric biomaterials towards nerve tissue engineering: a review. Artif Cells Nanomed Biotechnol. 2019;47(1):3524-3539. doi:10.1080/21691401.2019.1639723

45. Amani H, Arzaghi H, Bayandori M, et al. controlling cell behavior through the design of biomaterial surfaces: a focus on surface modification techniques. Adv Mater Interfaces. 2019;6(13):1900572. doi:10.1002/admi.v6.13

46. Mykhaylyk O, Sobisch T, Almstätter I, et al. Silica-iron oxide magnetic nanoparticles modified for gene delivery: a search for optimum and quantitative criteria. Pharm Res-Dordr. 2012;29(5):1344-1365. doi:10.1007/s11095-011-0661-9

47. Su L, Zhang B, Huang Y, Zhang H, Xu Q, Tan J. Superparamagnetic iron oxide nanoparticles modified with dimyristoylphosphatidylcholine and their distribution in the brain after injection in the rat substantia nigra. Mat Sci Eng. 2017;81:400-406. doi:10.1016/j. msec.2017.08.049

48. Lozano O, Mejia J, Tabarrant T, et al. Quantification of nanoparticles in aqueous food matrices using particle-induced X-ray emission. Anal Bioanal Chem. 2012;403(10):2835-2841. doi:10.1007/s00216-0125895-9

49. Lozano O, Mejia J, Masereel B, Toussaint O, Lison D, Lucas S. Development of a PIXE analysis method for the determination of the biopersistence of SiC and TiC nanoparticles in rat lungs. Nanotoxicology. 2011;6(3):263-271. doi:10.3109/17435390.2011.572301

50. Wang J, Xiang B, Deng J, et al. Externally applied static magnetic field enhances cardiac retention and functional benefit of magnetically iron-labeled adipose-derived stem cells in infarcted hearts. Stem Cell Transl Med. 2016;5(10):1380-1393. doi:10.5966/sctm.20150220

51. Muthana M, Scott SD, Farrow N, et al. A novel magnetic approach to enhance the efficacy of cell-based gene therapies. Gene Ther. 2008;15(12):902-910. doi:10.1038/gt.2008.57

52. Pu A, Stephenson EL, Yong VW. The extracellular matrix: focus on oligodendrocyte biology and targeting CSPGs for remyelination therapies. Glia. 2018;66(9):1809-1825. doi:10.1002/glia.v66.9

53. Zou JL, Sun JH, Qiu S, et al. Spatial distribution affects the role of CSPGs in nerve regeneration via the actin filament-mediated pathway. Exp Neurol. 2018;307:37-44. doi:10.1016/j.expneurol.2018.05.023

54. Raspa A, Bolla E, Cuscona C, Gelain F. Feasible stabilization of chondroitinase abc enables reduced astrogliosis in a chronic model of spinal cord injury. CNS Neurosci Ther. 2019;25(1):86-100. doi:10.1111/cns.2019.25.issue-1

55. Quraishe S, Forbes LH, Andrews MR. The extracellular environment of the CNS: influence on plasticity, sprouting, and axonal regeneration after spinal cord injury. Neural Plast. 2018;2018:2952386. doi: $10.1155 / 2018 / 2952386$ 


\section{Publish your work in this journal}

The International Journal of Nanomedicine is an international, peerreviewed journal focusing on the application of nanotechnology in diagnostics, therapeutics, and drug delivery systems throughout the biomedical field. This journal is indexed on PubMed Central, MedLine, CAS, SciSearch ${ }^{\circledR}$, Current Contents ${ }^{\circledR} /$ Clinical Medicine, $^{2}$
Journal Citation Reports/Science Edition, EMBase, Scopus and the Elsevier Bibliographic databases. The manuscript management system is completely online and includes a very quick and fair peer-review system, which is all easy to use. Visit http://www.dovepress.com/ testimonials.php to read real quotes from published authors.

Submit your manuscript here: https://www.dovepress.com/international-journal-of-nanomedicine-journal 